\title{
Recursive Algorithms for Pricing Discrete Variance options and Volatility Swaps under Time-changed Lévy Processes
}

\author{
WENDONG ZHENG, CHI HUNG YUEN \& YUE KUEN KWOK ${ }^{1}$ \\ Department of Mathematics, Hong Kong University of Science and Technology, Hong Kong
}

November 14, 2015

\begin{abstract}
We propose robust numerical algorithms for pricing variance options and volatility swaps on discrete realized variance under general time-changed Lévy processes. Since analytic pricing formulas of these derivatives are not available, some of the earlier pricing methods use the quadratic variation approximation for the discrete realized variance. While this approximation works quite well for long-maturity options on discrete realized variance, numerical accuracy deteriorates for options with low frequency of monitoring or short maturity. To circumvent these shortcomings, we construct numerical algorithms that rely on the computation of the Laplace transform of the discrete realized variance under time-changed Lévy processes. We adopt the randomization of the Laplace transform of the discrete log return with a standard normal random variable and develop a recursive quadrature algorithm to compute the Laplace transform of the discrete realized variance. Our pricing approach is rather computationally efficient when compared with the Monte Carlo simulation and works particularly well for discrete realized variance and volatility derivatives with low frequency of monitoring or short maturity. The pricing behaviors of variance options and volatility swaps under various time-changed Lévy processes are also investigated.
\end{abstract}

Keywords: time-changed Lévy processes, variance options, volatility swaps, discrete sampling

\section{Introduction}

The discrete realized variance of the log return of a risky stock is defined as the sum of the squared log returns of the stock price observed on a set of discrete monitoring dates. Variance swaps are forward contracts on the discrete realized variance of the price process of an underlying stock. These variance derivatives exhibit pure exposure to volatility when used in trading and risk management of volatility when compared to the use of conventional stock options. The pricing of variance and volatility derivatives under various models of the stock price dynamics has been well explored in the literature. The pricing approaches can be divided into two categories. The first category is the non-parametric approach that does not rely on a particular choice of the asset price model. The fair strike of a variance swap with continuous sampling can be obtained by the notion of replication of a continuum of stock options with varying strikes. This approach has been explained in the works by Neuberger (1994), Demeterfi et al. (1999) and Carr and Lee (2009). The shortcoming of this approach is that traded option prices are only available for a limited number of strikes while

\footnotetext{
${ }^{1}$ Correspondence author; email: maykwok@ust.hk; fax number: 852-2358-1643
} 
replication of the variance swap requires the market prices of options for a full spectrum of strikes.

In the model-dependent pricing approach, the quadratic variation of the continuous realized variance is commonly adopted as an approximation to its discrete counterpart since it provides good analytical tractability by virtue of an explicit representation of the associated characteristic function. Earlier research works that employ the quadratic variation approximation for pricing variance swaps have been performed under the Heston stochastic volatility model (Swishchuk, 2004), the 3/2-model (Carr and Sun, 2007) and the time-changed Lévy processes (Carr et al., 2012). For pricing of options on realized variance under various stochastic volatility models, one may refer to the papers by Carr et al. (2005) for pure jump processes, Sepp (2008) for an extended Heston model with simultaneous jumps in the underlying and variance processes (SVJJ model), and Kallsen et al. (2011) for general affine models with jumps. The quadratic variation approximation is seen not to perform well for short-maturity variance derivatives with non-linear payoffs, like the volatility swaps and options on discrete realized variance. Keller-Ressel and Muhle-Karbe (2013) propose an exact pricing formula for variance options under the Lévy models by randomizing the Laplace transform of the discrete log return with a standard normal variate.

Instead of adopting the quadratic variation approximation under the continuous realized variance, the recent papers deal directly with the contractual specification of discrete monitoring of the realized variance. Broadie and Jain (2008) and Zhu and Lian (2011) derive closed form formulas for pricing discrete variance swaps under the Heston model. Zheng and Kwok (2014a) consider pricing exotic variance swaps (including the gamma swaps and corridor swaps) under the stochastic volatility model with simultaneous jumps (SVJJ model). Itkin and Carr (2010) employ the forward characteristic function method to price variance swaps under the time-changed Lévy processes with the Cox-Ingersoll-Ross (CIR) process as the clock rate.

Beyond pricing of the variance swap products, other researchers have proposed various analytic approximation methods or numerical algorithms to price discrete variance derivatives with non-linear terminal payoff structures. Sepp (2012) approximates the discrete realized variance by the continuous realized variance with an additional correction term from a lognormal model in the SVJJ model. This method works well for options that are nearthe-money (see Drimus et al., 2014). Drimus et al. (2014) study the discretization errors on discrete variance options arising from continuous sampling approximation via the central limit theorem. They propose a simplified one-dimensional Monte Carlo simulation method and derive a set of analytic pricing formulas based on the asymptotic distribution. For low frequency of sampling of the discrete realized variance, their approximation method does not perform quite well due to poor approximation by the normal distribution using a few sampling points. Lian et al. (2014) approximate the characteristic function of the discrete realized variance in the stochastic volatility models and derive semi-analytic formulas for discrete variance derivatives. Zheng and Kwok (2014b) apply the saddlepoint approximation for pricing options on discrete realized variance. They also propose an enhanced simulation method for pricing the same class of products under a class of stochastic volatility models with jumps. Though their saddlepoint method approximation works well in general, the simulation method does not perform well for short-maturity put options and volatility swaps. In addition, Zheng and Kwok (2015) derive efficient and accurate analytic approximation formulas for pricing options on discrete realized variance under stochastic volatility models using the conditioning variable approach equipped with partially exact and bounded approximations. By adopting the conditional gamma distribution approximation based on some asymptotic behavior of the discrete realized variance of the underlying asset price process, 
they obtain approximate pricing formulas that achieve high level of numerical accuracy in option values, even for short-maturity options on discrete realized variance.

For the literature on the numerical algorithms for pricing discrete variance derivatives, Little and Pant (2001) compute the fair strike of discrete variance swaps under a local volatility model by the finite difference scheme. Windcliff et al. (2006) extend the Little-Pant algorithm by incorporating jumps in the stock price dynamics. Baldeaux (2012) proposes an exact simulation for the 3/2-model with some variance reduction technique by applying the Lie symmetry analysis. Zheng and Kwok (2014c) employ the Fourier time-stepping algorithm to price variance derivatives under the additive processes. They adopt the CONV method (Lord et al., 2008) in their fast Fourier transform algorithm. Though it is desirable to incorporate stochastic volatility in the underlying stock price dynamics, the direct extension of their numerical scheme to the time-changed Lévy processes or stochastic volatility models is computationally infeasible due to curse of dimensionality.

The recursive algorithms have been widely used to price various kind of options under the Lévy processes, like pricing discretely monitored Asian options by Fusai and Meucci (2008). Similar approach has been used by Sullivan (2000) and Fusai and Recchioni (2007) to price discrete barrier options. Yamazaki (2014) and Umezawa and Yamazaki (2015) employ a similar recursive algorithm to price path-dependent stock options under the time-changed Lévy processes.

In this paper, we extend the recursive algorithms to price discrete variance and volatility derivatives under the time-changed Lévy processes. We employ the randomization formula in a recursive quadrature algorithm to compute the moment generating function of the discrete realized variance. The explicit Laplace transform formula of the activity rate process essentially reduces the additional computational cost that arises from the extra state variable in the time-changed Lévy processes. This key step makes numerical pricing of discrete variance derivatives under the time-changed Lévy processes computationally feasible. We propose effective recursive algorithms to compute the moment generating function of the discrete variance under the time-changed Lévy processes. Our method works particularly well for variance and volatility derivatives with short-maturity and low sampling frequency. The pricing errors are small even with reasonably large number of recursive iterations in the numerical computation. In the first stage of the construction of our proposed algorithms, we assume independence between the underlying Lévy process and activity rate process. By randomizing the squared terms in the discrete realized variance formula with an independent standard normal variable, the sum of the squared returns can be expressed as linear terms under an expectation of the normal variable. This expectation is iterating on each monitoring dates and can be effectively computed in term of a transition matrix. Option prices and the fair strikes of discrete volatility swaps can be obtained through an inverse Laplace transform. To accommodate the leverage effect, we introduce a specific correlated diffusion term into the underlying Lévy process.

This paper is organized as follows. In the next section, we provide a brief description of the time-changed Lévy processes and their associated activity rate processes. The analytic formulas for the Laplace transform of the joint density of variance and integrated variance are derived. In Section 3, the general framework of our pricing approach is presented. The implementation of our numerical method is outlined in matrix forms. Numerical tests on numerical accuracy of our algorithms are presented in Section 4. Comparative analysis on different parameters are also studied in this section. Finally, we conclude our results in Section 5. 


\section{Time-changed Lévy processes}

Due to the stationary increment property, Lévy process is incapable of generating stochastic volatility effect. The time-changed Lévy processes provides a solution to the problem by introducing stochastic volatility through a random time change mechanism (Carr and Wu, 2004). Moreover, by manipulating the correlation between the base L'evy process and the stochastic activity rate process that models the time change, time-changed Lévy processes are able to generate the desired leverage effect.

\subsection{Lévy processes}

We start with a probability space $(\Omega, \mathscr{F}, \mathbb{Q})$ with a complete filtration $\mathcal{F}=\left\{\mathscr{F}_{t} \mid t \geq 0\right\}$, where $\mathbb{Q}$ is a risk neutral measure. Let the real-valued stochastic process $\left(X_{t}\right)_{t \geq 0}$ with $X_{0}=0$ be a Lévy process that is adapted to $\mathcal{F}$, with right continuous sample paths and left limits. Then, the characteristic function of $X_{t}$ admits the following Lévy-Khintchine representation (Bertoin, 1996):

$$
\phi_{X_{t}}(u)=\mathbb{E}\left[e^{i u X_{t}}\right]=e^{-t \psi_{X}(u)}, \quad t \geq 0,
$$

for $u \in \mathbb{R}$, where the characteristic exponent $\psi_{X}(u)$ is given by

$$
\psi_{X}(u)=-i \mu u+\frac{1}{2} \sigma^{2} u^{2}+\int_{\mathbb{R} \backslash\{0\}}\left(1-e^{i u x}+i u x \mathbf{1}_{|x|<1}\right) \Pi(\mathrm{d} x) .
$$

Here, the triplet $\left(\mu, \sigma^{2}, \Pi\right)$ is called the Lévy characteristics of $X$. The Lévy measure $\Pi$ is assumed to satisfy the finite quadratic variation condition: $\int_{\mathbb{R} \backslash\{0\}}\left(1 \wedge x^{2}\right) \Pi(\mathrm{d} x)<\infty$.

Jumps in the asset price processes have been frequently observed in the financial markets (Geman et al., 2001). Within a small time interval, there may be a lot of small jumps in the stock price movement. It may be difficult to distinguish if the contribution comes from the diffusion or jump component [see Aït-Sahalia (2004) for the methods of detecting jumps]. The financial models with compound Poisson jumps exhibit finite activity of the jump component, as in the Merton model (1976) and Kou model (2002). However, the Poisson process is incapable of capturing the high frequency of small jumps. Alternative Lévy type pure jump models with infinite activity have been proposed. These include the Variance Gamma (VG) model of Madan and Seneta (1990), the Normal Inverse Gaussian (NIG) model (BarndorffNislsen, 1998), the generalized hyperbolic model (Eberlein et al., 1998) and CGMY model (Carr et al., 2002). These models show better fit to market data. Besides, they admit explicit formulas of Lévy measures and characteristic exponents, which provides good tractability for financial modeling. In this paper, we will use these two processes as the base Lévy process.

1. The NIG process can be generated by subordinating a Brownian motion with drift by an independent inverse Gaussian process. The Lévy density of the NIG process is

$$
\nu_{\mathrm{NIG}}(x)=\frac{\delta \alpha}{\pi} \frac{e^{\beta x} K_{1}(\alpha|x|)}{|x|}
$$

where $\alpha>0,-\alpha<\beta<\alpha, \delta>0$ and $K_{\lambda}(x)$ denotes the modified Bessel function of the third kind with index $\lambda$. The characteristic exponent is given by

$$
\psi_{\mathrm{NIG}}(u)=\delta\left(\sqrt{\alpha^{2}-(\beta+i u)^{2}}-\sqrt{\alpha^{2}-\beta^{2}}\right) .
$$


2. The VG process can be obtained by subordinating a Brownian motion by an independent gamma process. It has the following Lévy measure:

$$
\nu_{\mathrm{VG}}(x)=\left\{\begin{array}{ll}
\frac{C \exp (G x)}{|x|} & x<0 \\
\frac{C \exp (-M x)}{x} & x>0
\end{array},\right.
$$

where $C, G$ and $M$ are positive real parameters. The characteristic exponent is given by

$$
\psi_{\mathrm{VG}}(u)=-C \ln \left(\frac{G M}{G M+(M-G) i u+u^{2}}\right) .
$$

\subsection{Activity rate processes}

Let $T=\left(T_{t}\right)_{t \geq 0}$ be a right-continuous process with left limits. For each $t, T_{t}$ is a stopping time with respect to $\left(\mathscr{F}_{t}\right)_{t \geq 0}$. We further assume that $T_{t}$ is finite $\mathbb{Q}$-a.s. for all $t \geq 0$ and goes to infinity as $t \rightarrow \infty$. Then, a time-changed Lévy process $Y=\left(Y_{t}\right)_{t \geq 0}$ is defined by subordinating $X_{t}$ with $T_{t}$ as follows:

$$
Y_{t}=X_{T_{t}}
$$

The typical approach of specifying the random time process $T$ is through a non-negative instantaneous activity rate process $\left(v_{t}\right)_{t \geq 0}$, where

$$
T_{t}=\int_{0}^{t} v_{s} \mathrm{~d} s .
$$

The stochastic time changes can be applied separately to the jump component and diffusion component to generate stochastic volatility arising from different sources (Huang and $\mathrm{Wu}, 2004$; Carr and $\mathrm{Wu}, 2004)$. The correlation between the time change process and the underlying Lévy process can be embedded into the model to represent the leverage effect in various ways (Carr and $\mathrm{Wu}, 2004$ ).

There is a large set of processes that can be used as the activity rate process. In practice, we restrict the candidate processes to be some well behaved processes in the sense that they admit good tractability and financial interpretation. To facilitate the numerical recursive algorithm proposed in the paper, we require that the joint distribution of $v_{t}$ and $\int_{0}^{t} v_{s} \mathrm{~d} s$ can be analytically characterized by either an explicit joint characteristic function or an explicit Laplace transform of the joint density with respect to $\int_{0}^{t} v_{s} \mathrm{~d} s$. A few examples of the activity rate process are provided as follows.

\section{CIR process}

The Cox-Ingersoll-Rox (CIR) process $v_{t}$ is a Markovian process satisfying the following stochastic differential equation (SDE)

$$
\mathrm{d} v_{t}=k\left(\theta-v_{t}\right) \mathrm{d} t+\epsilon \sqrt{v_{t}} \mathrm{~d} W_{t}^{v}
$$

where $W_{t}^{v}$ is a Brownian motion and $k, \theta, \epsilon$ are positive constants which are assumed to satisfy the Feller condition $2 k \theta \geq \epsilon^{2}$. Let $f_{\mathrm{CIR}}\left(\tau, v, y ; v_{0}\right)$ be the joint transition density of $v_{\tau}$ and $\int_{0}^{\tau} v_{s} \mathrm{~d} s$ from $\left(v_{0}, 0\right)$ at time 0 to $(v, y)$ at time $\tau$. The following lemma gives the Laplace transform of the joint density $f_{\mathrm{CIR}}\left(\tau, v, y ; v_{0}\right)$ with respect to $y$. 
Lemma 1 For the CIR process defined in eq. (2.2), the Laplace transform $G_{C I R}\left(\tau, v, \eta ; v_{0}\right)$ of the joint density $f_{C I R}\left(\tau, v, y ; v_{0}\right)$ with respect to $y$ is given by

$$
\begin{aligned}
G_{C I R}\left(\tau, v, \eta ; v_{0}\right)= & \int_{0}^{\infty} e^{-\eta y} f_{C I R}\left(\tau, v, y ; v_{0}\right) \mathrm{d} y \\
= & 2 \exp \left(\epsilon^{2} k\left(v_{0}-v\right)-\frac{\left(1+e^{\delta} \tau\right)\left(v+v_{0}\right) \delta \epsilon^{2}}{e^{\delta \tau}-1}\right)\left[\frac{e^{-\delta \tau}\left(e^{\delta \tau}-1\right)^{2} v \epsilon^{4}}{v_{0} \delta^{2}}\right]^{\frac{\beta-1}{2}} \\
& {\left[\frac{e^{\frac{1}{2}(k+\delta) \tau} \delta}{\left(e^{\delta \tau}-1\right) \epsilon^{2}}\right]^{\beta} I_{\beta-1}\left(4 \sqrt{\frac{e^{\delta \tau} v_{0} v \delta^{2}}{\left(e^{\delta \tau}-1\right)^{2} \epsilon^{4}}}\right) }
\end{aligned}
$$

where

$$
\delta=\sqrt{k^{2}+2 \epsilon^{2} \eta} \text { and } \beta=\frac{2 k \theta}{\epsilon^{2}} .
$$

Note that $I_{\nu}$ is the modified Bessel function of the first kind of order $\nu$

$$
I_{\nu}(z)=\left(\frac{z}{2}\right)^{\nu} \sum_{k=0}^{\infty} \frac{\left(\frac{z^{2}}{2}\right)^{k}}{k ! \Gamma(\nu+k+1)} .
$$

The proof of Lemma 1 is presented in the Appendix A.

\section{3/2-process}

Another appropriate choice of the activity rate process is the $3 / 2$-process $v_{t}$ that is defined by

$$
\mathrm{d} v_{t}=v_{t}\left(p-q v_{t}\right) \mathrm{d} t+\sigma v_{t}^{3 / 2} \mathrm{~d} W_{t}^{v},
$$

where $W_{t}^{v}$ is a Brownian motion and $p, q, \sigma$ are positive constants. Its sample paths exhibit a more volatile structure than that of the CIR process. We let $f_{3 / 2}\left(\tau, v, y ; v_{0}\right)$ be the corresponding joint transition density of $v_{\tau}$ and $\int_{0}^{\tau} v_{s} \mathrm{~d} s$ at time $\tau$ given $v_{0}$ at time 0 for the 3/2-process. The Laplace transform of the joint density is presented in Lemma 2.

Lemma 2 For the 3/2-process defined in eq. (2.4), the Laplace transform $G_{3 / 2}\left(\tau, v, \eta ; v_{0}\right)$ of the joint density $f_{3 / 2}\left(\tau, v, y ; v_{0}\right)$ with respect to $y$ is given by (Zheng and Zeng, 2014)

$$
\begin{aligned}
G_{3 / 2}\left(\tau, v, \eta ; v_{0}\right) & =\int_{0}^{\infty} e^{-\eta y} f_{3 / 2}\left(\tau, v, y ; v_{0}\right) \mathrm{d} y \\
& =\frac{A_{\tau}}{C_{\tau} v^{2}} \exp \left(-\frac{A_{\tau} v_{0}+v}{C_{\tau} v_{0} v}\right)\left(\frac{A_{\tau} v_{0}}{v}\right)^{\frac{1}{2}+\frac{q}{\sigma^{2}}} I_{2 c}\left(2 C_{\tau}^{-1} \sqrt{\frac{A_{\tau}}{v v_{0}}}\right)
\end{aligned}
$$

where

$$
c=\sqrt{\left(\frac{1}{2}+\frac{q}{\sigma^{2}}\right)^{2}+\frac{2 \eta}{\sigma^{2}}}, A_{\tau}=e^{p \tau}, \quad C_{\tau}=\frac{\sigma^{2}\left(e^{p \tau}-1\right)}{2 p} .
$$

\section{Quadratic Ornstein-Uhlenbeck process}

The third choice of the activity rate process is the quadratic Ornstein-Uhlenbeck (OU) process which is given by

$$
v_{t}=\left(z_{t}+p+q t\right)^{2},
$$

where $p, q$ are two constants, and $z_{t}$ is an OU process defined by

$$
\mathrm{d} z_{t}=\kappa\left(\theta-z_{t}\right) \mathrm{d} t+\sigma \mathrm{d} L_{t}
$$


Here, $L_{t}$ is a Lévy process. When $L_{t}$ is a Brownian motion, $v_{t}$ is called a quadratic Gaussian OU process. For a quadratic Gaussian OU process, the joint characteristic function has an exponential quadratic form, where

$$
\begin{aligned}
\phi(\lambda, \eta) & =\mathbb{E}\left[\exp \left(\lambda v_{s}+\eta \int_{t}^{s} v_{u} d u\right) \mid \mathscr{G}_{t}\right] \\
& =\exp \left(H_{0}(t, s ; \lambda, \eta)+H_{1}(t, s ; \lambda, \eta) z_{t}+H_{2}(t, s ; \lambda, \eta) z_{t}^{2}\right)
\end{aligned}
$$

Here, $\mathcal{G}=\left\{\mathscr{G}_{t} \mid t \geq 0\right\}$ is the natural filtration generated by $\left(v_{t}\right)_{t \geq 0}$. The parameter functions $H_{0}, H_{1}$ and $H_{2}$ are determined by solving a Riccati system of ordinary differential equations. As shown in Appendix B, while we manage to find the parameter functions explicitly, the complexity of the mathematical expressions retrains us from performing the inverse Laplace transform with respect to $\lambda$ to obtain a closed form formula for $G_{q o u}$, like the results shown in Lemma 1 or Lemma 2. One has to resort to numerical procedure to find the inverse Laplace transform.

\subsection{Stock price process}

We assume that the stock price process follows an exponential time-changed Lévy process defined as

$$
\ln \frac{S_{t}}{S_{0}}=(r-d) t+X_{T_{t}}-\varphi(1) T_{t},
$$

where $\varphi(u)=-\psi(-i u)$ is the cumulant exponent of $X_{t}$, and $\varphi(1) T_{t}$ is the mean adjustment term so that the discounted stock price satisfies the martingale condition. By changing the drift coefficient $\mu$ of the base Lévy process to be $\mu-\varphi(1)$, we can absorb the mean adjustment term into the process and rewrite the stock price process as (Carr and Wu, 2004)

$$
\ln \frac{S_{t}}{S_{0}}=(r-d) t+\widetilde{X}_{T_{t}}
$$

where $\widetilde{X}$ is the mean adjusted base Lévy process. For notational convenience, we always assume $X$ to be mean adjusted hereafter. In the following section, we first derive our numerical algorithm by assuming zero correlation between $X$ and $T$, and then extend our approach to accommodate the leverage effect.

\section{Formulation of the recursive algorithm}

First, we present the product nature of various discrete variance derivatives, including the volatility swaps and options on variance. We then show how to perform numerical computation of the Laplace transform of the discrete realized variance of the stock price process under the time-changed Lévy processes.

\subsection{Discrete variance and volatility derivatives}

We consider the tenor of the discrete realized variance to be $[0, \mathcal{T}]$ with monitoring dates $0=t_{0}<t_{1}<\cdots<t_{N}=\mathcal{T}$, where $\mathcal{T}$ is the maturity date and $N$ is the total number of monitoring dates. We use $V_{d}$ to denote the discrete realized variance over $\left[t_{0}, t_{N}\right]$ defined by

$$
V_{d}=\frac{F_{A}}{N} \sum_{k=1}^{N}\left(\ln \frac{S_{t_{k}}}{S_{t_{k-1}}}\right)^{2},
$$


where $F_{A}$ is the annualized factor. We take $F_{A}=12$ for monthly monitoring and $F_{A}=52$ for weekly monitoring. Let $\triangle t_{k}$ denote the time interval between $t_{k-1}$ and $t_{k}, k=1,2, \cdots, N$.

1. A discrete variance swap is a forward contract that exchanges the discrete realized variance with a fixed strike. The fair strike $K_{\text {var }}$ of the variance swap gives zero value of the contract to both counterparties at initiation. It is given by the risk neutral expectation of $V_{d}$, where

$$
K_{\text {var }}=\mathbb{E}\left[V_{d}\right]
$$

2. A discrete volatility swap is a contract similar to the discrete variance swap. The holder can swap the square root of the discrete realized variance for a fixed strike. Its fair strike is given by

$$
K_{v o l}=\mathbb{E}\left[\sqrt{V_{d}}\right]
$$

3. The option on discrete realized variance gives the holder the right but not the obligation to exchange the discrete realized variance for a fixed strike. The undiscounted price $V_{p}$ of a put option with strike $K$ is given by

$$
V_{p}=\mathbb{E}\left[\left(K-V_{d}\right)^{+}\right] .
$$

Once we have computed the value of a put option, the value of the call option counterpart can be easily obtained by the call-put parity formula. The fair strike formula for the discrete variance swap under time-changed Lévy processes has been derived in Itkin and Carr (2010). However, they resort to analytic approximation when they compute the fair values of the options on variance and volatility swaps. As stated in Bühler (2006), such an approximation is not quite accurate for short-maturity variance derivatives with a small number of monitoring dates. In this paper, we consider numerical pricing of various types of discrete variance derivatives without any analytic approximation.

\subsection{Laplace transform of discrete realized variance}

To price discrete variance derivatives, we first compute the Laplace transform of the discrete realized variance of the stock price. For simplicity of notation, we write $S_{k}$ as $S_{t_{k}}, v_{k}$ as $v_{t_{k}}$, and $X_{T_{k}}$ as $X_{T_{t_{k}}}$, and let

$$
R_{k}=\ln \frac{S_{k}}{S_{k-1}}=(r-d) \Delta t_{k}+X_{T_{k}}-X_{T_{k-1}} \quad \text { and } \quad I_{N}=\sum_{k=1}^{N} R_{k}^{2} .
$$

We write the Laplace transform of $I_{N}$ and $R_{k}^{2}$ as

$$
\Psi_{I_{N}}(\lambda)=\mathbb{E}\left[e^{-\lambda I_{N}}\right] \quad \text { and } \quad \Phi_{k}(\lambda)=\mathbb{E}\left[e^{-\lambda R_{k}^{2}}\right], \lambda \geq 0 .
$$

We first consider the trivial case where $T_{t}=t$. By virtue of the independent increment property of the base Lévy process, we have

$$
\Psi_{I_{N}}(\lambda)=\prod_{k=1}^{N} \Phi_{k}(\lambda)
$$

To deal with the Laplace transform of the squared process, Keller-Ressel and Muhle-Karbe (2013) propose the following randomization for an arbitrary Lévy process $X_{t}$ :

$$
\mathbb{E}\left[e^{-\lambda X_{t}^{2}}\right]=\mathbb{E}\left[e^{-t\left(\psi_{X}(Z \sqrt{2 \lambda})\right)}\right],
$$


where $Z$ is an independent standard normal random variable and the domain of $\lambda$ is extended to the entire positive half plane $\{\lambda \in \mathbb{C}: \operatorname{Re} \lambda \geq 0\}$. As a result, the Laplace transform of the squared process is reduced to an integral involving the normal density function:

$$
\mathbb{E}\left[e^{-\lambda X_{t}^{2}}\right]=\frac{1}{\sqrt{2 \pi}} \int_{-\infty}^{\infty} e^{-t \psi_{X}(z \sqrt{2 \lambda})} e^{-z^{2} / 2} \mathrm{~d} z
$$

It follows that

$$
\Phi_{k}(\lambda)=\frac{1}{\sqrt{2 \pi}} \int_{-\infty}^{\infty} e^{\Delta t_{k}(r-d) i x \sqrt{2 \lambda}-\Delta t_{k}\left(\psi_{X}(x \sqrt{2 \lambda})\right)-x^{2} / 2} \mathrm{~d} x
$$

\section{Independent time change}

To extend the above analytic representation to time-changed Lévy processes, we assume the activity rate process $\left(v_{t}\right)_{0 \leq t \leq \mathcal{T}}$ to be independent of the base Lévy process and let $\left(\mathscr{G}_{t}\right)_{t \geq 0}$ be the natural filtration generated by the activity rate process $\left(v_{t}\right)_{0 \leq t \leq \mathcal{T}}$. It is then straightforward to observe that the conditional Laplace transform $\Phi_{k \mid \mathscr{G}_{\mathcal{T}}}(\lambda)=\mathbb{E}\left[e^{\left.-\lambda R_{k}^{2} \mid \mathscr{G}_{\mathcal{T}}\right]}\right.$ admits

$$
\Phi_{k \mid G_{\mathcal{T}}}(\lambda)=\frac{1}{\sqrt{2 \pi}} \int_{-\infty}^{\infty} e^{\Delta t_{k}(r-d) i x \sqrt{2 \lambda}-\psi_{X}(x \sqrt{2 \lambda})\left(\int_{t_{k-1}}^{t_{k}} v_{s} \mathrm{~d} s\right)-x^{2} / 2} \mathrm{~d} x .
$$

To recover the unconditional Laplace transform, $\Psi_{I_{N}}(\lambda)$, we take the expectation with respect to the joint distribution of $\int_{t_{k-1}}^{t_{k}} v_{s} \mathrm{~d} s, k=1,2, \ldots, N$ [see eq. (3.3)]:

$$
\Psi_{I_{N}}(\lambda)=\mathbb{E}\left[\prod_{k=1}^{N} \Phi_{k \mid \mathscr{G}_{\mathcal{T}}}(\lambda)\right]
$$

Define the sequence of functions

$$
f_{k}(\lambda, y)=\frac{1}{\sqrt{2 \pi}} \int_{-\infty}^{\infty} e^{(r-d) \Delta t_{k} i x \sqrt{2 \lambda}-\psi_{X}(x \sqrt{2 \lambda}) y-x^{2} / 2} \mathrm{~d} x, \quad y \geq 0, k=1,2, \cdots, N
$$

In view of the Markovian property of the activity rate process, eq. (3.6) can be expressed as

$$
\Psi_{I_{N}}(\lambda)=\mathbb{E}\left[f_{1}\left(\lambda, \int_{t_{0}}^{t_{1}} v_{s} \mathrm{~d} s\right) \mathbb{E}\left[f_{2}\left(\lambda, \int_{t_{1}}^{t_{2}} v_{s} \mathrm{~d} s\right) \ldots \mathbb{E}\left[f_{N}\left(\lambda, \int_{t_{N-1}}^{t_{N}} v_{s} \mathrm{~d} s\right) \mid v_{N-1}\right] \ldots \mid v_{1}\right] \mid v_{0}\right] .
$$

Starting from the innermost expectation, it is easy to see that the computation of the above expression can be formulated by the following recursive procedure.

Proposition 3 Let $g_{k}(\lambda, v)$ be the conditional expectation by time $t_{k}$ which is defined by

$$
\begin{aligned}
g_{k}(\lambda, v) & =\mathbb{E}\left[g_{k+1}\left(\lambda, v_{k+1}\right) f_{k+1}\left(\lambda, \int_{t_{k}}^{t_{k+1}} v_{s} \mathrm{~d} s\right) \mid v_{k}=v\right], \quad k=0,1, \ldots, N-1 \\
g_{N}(\lambda, v) & =1, \quad \text { for all } v .
\end{aligned}
$$

The unconditional Laplace transform of $I_{N}$ is then given by

$$
\Psi_{I_{N}}(\lambda)=g_{0}\left(\lambda, v_{0}\right)
$$


Let $p\left(\tau, v_{k}, y ; v\right)$ be the joint transition density of $\left(v_{k}, \int_{t_{k-1}}^{t_{k}} v_{s} \mathrm{~d} s\right)$ from $(v, 0)$ at time $t_{k-1}$ to $\left(v_{k}, y\right)$ at time $t_{k}$ and $G\left(\tau, v_{k}, \eta ; v\right)=\int_{0}^{\infty} e^{-\eta y} p\left(\tau, v_{k}, y ; v\right) \mathrm{d} y$. Then, eq. (3.7) can be further written as

$$
\begin{aligned}
g_{k}(\lambda, v) & =\int_{0}^{\infty} \int_{0}^{\infty} \frac{g_{k+1}\left(\lambda, v_{k}\right)}{\sqrt{2 \pi}}\left[\int_{-\infty}^{\infty} e^{(r-d) \Delta t_{k} i x \sqrt{2 \lambda}-\psi_{X}(x \sqrt{2 \lambda}) y-x^{2} / 2} \mathrm{~d} x\right] p\left(\Delta t_{k}, v_{k}, y ; v\right) \mathrm{d} y \mathrm{~d} v_{k} \\
& =\int_{0}^{\infty} \frac{g_{k+1}\left(\lambda, v_{k}\right)}{\sqrt{2 \pi}} \int_{-\infty}^{\infty} e^{(r-d) \Delta t_{k} i x \sqrt{2 \lambda}-x^{2} / 2} G\left(\Delta t_{k}, v_{k}, \psi_{X}(x \sqrt{2 \lambda}) ; v\right) \mathrm{d} x \mathrm{~d} v_{k} .
\end{aligned}
$$

The inner integral in eq. (3.8) can be efficiently calculated if $G$ is known explicitly. This is true when $v_{t}$ is modeled as a CIR or $3 / 2$ process. For other processes, such as the quadratic Gaussian OU process, which admit an explicit joint characteristic function, an additional numerical inverse Laplace transform is needed to obtain the values of $G$. Also, by virtue of the Gaussian kernel which exhibits a fast decaying rate, the computation of the inner integral with respect to $x$ does not involve any numerical difficulties.

\section{Leverage effect}

The numerical procedure discussed in the previous subsection relies on the assumption of zero correlation, which enables us to disentangle the time change process from the base Lévy process. The algorithm no longer works once we introduce correlation in the usual way, such as a correlated diffusion part. Fortunately, our recursive algorithm can be easily extended to the following alternative approach of generating correlation. Based on the model in Kallsen et al. (2011), we consider

$$
\ln \frac{S_{t}}{S_{0}}=(r-d) t+X_{T_{t}}+\varrho \int_{0}^{t} \sqrt{v_{s}} \mathrm{~d} W_{s}^{v}-\frac{\varrho^{2}}{2} \int_{0}^{t} v_{s} \mathrm{~d} s
$$

where $W_{s}^{v}$ is the same Brownian motion that drives the activity process $v_{t}$. In eq. (3.9), while we maintain the independency between $X_{t}$ and $T_{t}$, we introduce leverage effect by directly appending a correlated diffusion part (with a mean corrector) to the underlying stock price process. The modified process can still be viewed as a time-changed Lévy process, as the canonical time changing theory guarantees that there exists a Brownian motion $B_{t}$ such that the time changed process $B_{\int_{0}^{t} v_{s} \mathrm{~d} s}$ has the same distribution of $\int_{0}^{t} \sqrt{v_{s}} \mathrm{~d} W_{s}^{v}$ (Barndorff-Nielsen and Shiriaev, 2010).

It is worth noting that most prevailing stochastic volatility models are special cases of the process specified by eq. (3.9). For instance, consider the following Heston model dynamics with stochastic variance $V_{t}$ :

$$
\ln \frac{S_{t}}{S_{0}}=(r-d) t-\frac{1}{2} \int_{0}^{t} V_{s} \mathrm{~d} s+\sqrt{1-\rho^{2}} \int_{0}^{t} \sqrt{V_{s}} \mathrm{~d} W_{s}+\rho \int_{0}^{t} \sqrt{V_{s}} \mathrm{~d} W_{s}^{V}
$$

where $W_{t}^{V}$ is the Brownian motion that drives the variance process and $W_{t}$ is another Brownian motion that is independent of $W_{t}^{V}$. Apparently, it is seen as a specialization of eq. (3.9) with $X$ being a Lévy process with characteristics $\left(-\frac{1}{2}\left(1-\rho^{2}\right), 1-\rho^{2}, 0\right), T_{t}=\int_{0}^{t} V_{s} \mathrm{~d} s$, and $\varrho=\rho$.

In order that our recursive algorithm works in the presence of correlation in the form of eq. (3.9), the activity rate process $v_{t}$ has to satisfy several technical constraints. Consider a diffusion process of the following general form:

$$
\mathrm{d} v_{t}=\alpha\left(t, v_{t}\right) \mathrm{d} t+\beta\left(t, v_{t}\right) \mathrm{d} W_{t}^{v}
$$


where $\alpha\left(t, v_{t}\right)$ and $\beta\left(t, v_{t}\right)$ are deterministic functions of $v_{t}$, such that $\beta(\cdot, \cdot)$ is nonzero and differentiable over $[0, \mathcal{T}] \times(0, \infty)$. Define

$$
P(t, y)=\int_{0}^{y} \frac{\sqrt{z}}{\beta(t, z)} \mathrm{d} z, \quad \text { and } \quad Q(t, y)=\frac{\partial P}{\partial t}+\alpha(t, y) \frac{\partial P}{\partial y}+\frac{1}{2} \beta^{2}(t, y) \frac{\partial^{2} P}{\partial y^{2}} .
$$

Suppose that there exist a constant $a$ and a deterministic and integrable function $b(t)$ such that

$$
Q(t, y)=a y+b(t), \text { for all } y>0,0 \leq t \leq \mathcal{T} .
$$

The recursive algorithm can still be used to compute the Laplace transform of the discrete realized variance under the model specification given by eq. (3.9).

To see this, it can be easily verified by Itô's Lemma that

$$
\mathrm{d} P\left(t, v_{t}\right)=Q\left(t, v_{t}\right) \mathrm{d} t+\sqrt{v_{t}} \mathrm{~d} W_{t}^{v}=\left[a v_{t}+b(t)\right] \mathrm{d} t+\sqrt{v_{t}} \mathrm{~d} W_{t}^{v} .
$$

It then follows that

$$
\int_{t_{k-1}}^{t_{k}} \sqrt{v_{s}} \mathrm{~d} W_{s}^{v}=P\left(v_{t_{k}}\right)-P\left(v_{t_{k-1}}\right)-a \int_{t_{k-1}}^{t_{k}} v_{s} \mathrm{~d} s-\int_{t_{k-1}}^{t_{k}} b_{s} \mathrm{~d} s .
$$

As a result, the conditional Laplace transform of $R_{k}$ can now be written as

$$
\begin{aligned}
\mathbb{E}\left[e^{u R_{k}} \mid \mathscr{G}_{\mathcal{T}}\right] & =\mathbb{E}\left[\exp \left(u\left((r-d) \Delta t_{k}+X_{T_{k}}-X_{T_{k-1}}+\varrho \int_{t_{k-1}}^{t_{k}} \sqrt{v_{s}} \mathrm{~d} W_{s}^{v}-\frac{\varrho^{2}}{2} \int_{t_{k}}^{t_{k+1}} v_{s} \mathrm{~d} s\right)\right)\right] \\
& =\exp \left(u \int_{t_{k-1}}^{t_{k}} c_{s} \mathrm{~d} s+\varrho u\left[P\left(v_{t_{k}}\right)-P\left(v_{t_{k-1}}\right)\right]+\xi(u) \int_{t_{k-1}}^{t_{k}} v_{s} \mathrm{~d} s\right),
\end{aligned}
$$

where $c_{t}=r-d-\varrho b_{t}$ and $\xi(u)=-\psi_{X}(-i u)-a \varrho u-\varrho^{2} u / 2$. Using the randomization method in eq. (3.4), we can derive the conditional Laplace transform of $R_{k}^{2}$ as

$$
\begin{aligned}
\Phi_{k \mid \mathscr{G}_{\mathcal{T}}}(\lambda)=\frac{1}{\sqrt{2 \pi}} \int_{-\infty}^{\infty} \exp (i x & \sqrt{2 \lambda} \int_{t_{k-1}}^{t_{k}} c_{s} \mathrm{~d} s+\xi(i x \sqrt{2 \lambda}) \int_{t_{k-1}}^{t_{k}} v_{s} \mathrm{~d} s \\
& \left.+i \varrho x \sqrt{2 \lambda}\left[P\left(v_{t_{k}}\right)-P\left(v_{t_{k-1}}\right)\right]-x^{2} / 2\right) \mathrm{d} x .
\end{aligned}
$$

Finally, the new recursive formula for $g_{k}(\lambda, v)$ is obtained as follows:

$$
\begin{aligned}
g_{k}(\lambda, v)= & \int_{0}^{\infty} \frac{g_{k+1}\left(\lambda, v_{k}\right)}{\sqrt{2 \pi}} \int_{-\infty}^{\infty} G\left(\Delta t_{k}, v_{k},-\xi(i x \sqrt{2 \lambda}) ; v\right) \\
& \exp \left(i x \sqrt{2 \lambda} \int_{t_{k-1}}^{t_{k}} c_{s} \mathrm{~d} s+i \varrho x \sqrt{2 \lambda}\left[P\left(v_{t_{k}}\right)-P\left(v_{t_{k-1}}\right)\right]-x^{2} / 2\right) \mathrm{d} x \mathrm{~d} v_{k} .
\end{aligned}
$$

As a remark, it can be easily shown that the assumption (3.11) holds for the most popular stock price models. For instance, for the CIR process defined in eq. (2.2), we find that

$$
P\left(v_{t}\right)=\frac{v_{t}}{\epsilon} \quad \text { and } \quad Q\left(v_{t}\right)=\frac{k\left(\theta-v_{t}\right)}{\epsilon} .
$$

For the 3/2-process defined in eq. (2.4), the corresponding $P\left(v_{t}\right)$ and $Q\left(v_{t}\right)$ are found to be

$$
P\left(v_{t}\right)=\frac{\ln v_{t}}{\sigma} \text { and } Q\left(v_{t}\right)=-\left(\frac{q}{\sigma}+\frac{\sigma}{2}\right) v_{t}+\frac{p}{\sigma} .
$$

As for the quadratic Gaussian OU process defined by eq. (2.6a), the recursive algorithm in general does not work for the extended stock price dynamics specified in eq. (3.9). However, if we let $q=0$ and $p=-\theta$, it can easily verified that $v_{t}$ satisfies the following SDE:

$$
\mathrm{d} v_{t}=\left(\sigma^{2}-2 \kappa v_{t}\right) \mathrm{d} t+2 \sigma \sqrt{v_{t}} \mathrm{~d} W_{t}
$$

which satisfies the assumption (3.11). 


\section{Vectorized implementation}

It is convenient to rewrite our computational formula in a vectorized form so that the recursive iteration can take full advantage of the efficiency of matrix multiplication. We choose the $v$-grid as a $M$-point vector $\mathbf{v}=\left\{v_{m}\right\}_{m=1}^{M} \in \mathbb{R}^{M}$. We now discretize eq. (3.8) as follows

$$
g_{k}\left(\lambda, v_{l}\right)=\sum_{m=1}^{M} w_{m} \frac{g_{k+1}\left(\lambda, v_{m}\right)}{\sqrt{2 \pi}}\left[\int_{-\infty}^{\infty} e^{(r-d) \Delta t_{k} i x \sqrt{2 \lambda}-x^{2} / 2} G\left(\Delta t_{k}, v_{m}, \psi_{X}(x \sqrt{2 \lambda}) ; v_{l}\right) \mathrm{d} x\right] \text {, }
$$

where $\mathbf{w}=\left\{w_{m}\right\}_{m=1}^{M} \in \mathbb{R}^{M}$ is a weight vector associated with the numerical quadrature rule. Various numerical integration methods, like the trapezoidal rule or Gaussian quadrature rule, can be implemented in our recursive algorithm by choosing a combination of grid points of $\mathbf{v}$ and $\mathbf{w}$. Suppose the time interval is uniformly given by $\Delta t$. For each $\lambda$, we define a matrix $H(\lambda)$ whose entry at the $l$ th row and $m$ th column is given by

$$
h_{l, m}(\lambda)=\frac{1}{\sqrt{2 \pi}} \int_{-\infty}^{\infty} e^{(r-d) \Delta t i x \sqrt{2 \lambda}-x^{2} / 2} G\left(\Delta t, v_{m}, \psi_{X}(x \sqrt{2 \lambda}) ; v_{l}\right) \mathrm{d} x
$$

Obviously, $H$ can be considered as a stationary transition matrix $H$ for $\mathbf{v}$. As a remark, provided that $G$ admits a closed-form representation, the computation of $h_{l, m}(\lambda)$ amounts to numerical evaluation of an one-dimensional integral. However, suppose only the joint density or the joint characteristic function is expressible in an analytic form, then an extra procedure of numerical evaluation of the Laplace transform or inverse Laplace transform is required for computing $h_{l, m}(\lambda)$.

The recursive algorithm can be presented succinctly in matrix notation as follows

$$
g_{k}(\lambda, \mathbf{v})=H(\lambda) \operatorname{diag}(\mathbf{w}) g_{k+1}(\lambda, \mathbf{v}),
$$

with $g_{N}(\lambda, \mathbf{v})=(1, \ldots, 1)^{\mathrm{T}} \in \mathbb{R}^{M}$, where $\operatorname{diag}(\mathbf{w})$ denotes the diagonal matrix whose diagonal elements are given by the vector $\mathbf{w}$. This formula essentially reduces the recursive iteration procedure to a $N$-folded matrix multiplication. As a result, the Laplace transform $\Psi_{I_{N}}(\lambda)$ can be expressed as

$$
g_{0}(\lambda, \mathbf{v})=[H(\lambda) \operatorname{diag}(\mathbf{w})]^{N}(1, \ldots, 1)^{\mathrm{T}} .
$$

When the initial value $v_{0}$ of the activity rate process is not chosen as a nodal point, an interpolation procedure can be applied to obtain $g_{0}\left(\lambda, v_{0}\right)$.

An interesting feature of our recursive algorithm is that the computational complexity of calculating $g_{0}(\lambda, \mathbf{v})$ is independent of the number of monitoring instants $N$. The clue lies in the Cholesky decomposition of the product matrix $H(\lambda) \operatorname{diag}(\mathbf{w})$, which is of order $O\left(M^{3}\right)$. After the decomposition, the power $N$ goes to the resultant diagonal matrix. The remaining matrix multiplication costs nothing more than $O\left(M^{3}\right)$. Suppose we use an $L$-point quadrature rule to compute $h_{l, m}$, then preparing matrix $H$ requires $O\left(L M^{2}\right)$. Due to the fast decay of the integrand of $H$, we typically have $L<M$. As a result, the overall computational complexity of calculating $g_{0}(\lambda, \mathbf{v})$ is still $O\left(M^{3}\right)$. Though $N$ has no direct impact on the computational complexity, we would need a denser grid $\mathbf{v}$ to maintain the same level of accuracy when $N$ increases. In actual numerical implementation, the computational costs do increase. 


\subsection{Derivative pricing formulas}

Given the Laplace transform of the discrete realized variance, the variance option prices and fair strikes of volatility swaps can be derived by using the transform method. Various earlier research papers have discussed different effective numerical schemes to evaluate the inverse Laplace transform (Kessel-Ressel and Muhle-Karbe, 2013). The transform method in pricing discrete variance derivatives based on our numerical method is demonstrated below.

The undiscounted value of a put option on discrete realized variance defined in eq. (3.2c) with strike $K$ is given by the following formula:

$$
\begin{aligned}
\mathbb{E}\left[\left(K-V_{d}\right)^{+}\right] & =\frac{F_{A}}{N} \mathbb{E}\left[\left(K^{\prime}-I_{N}\right)^{+}\right] \\
& =\frac{F_{A}}{N} \frac{1}{\pi} \int_{0}^{\infty} \operatorname{Re}\left[\frac{e^{K^{\prime}\left(\lambda_{r}+i \lambda\right)}}{\left(\lambda_{r}+i \lambda\right)^{2}} \Psi_{I_{N}}\left(\lambda_{r}+i \lambda\right)\right] \mathrm{d} \lambda \\
& \approx \frac{F_{A}}{N} \frac{1}{\pi} \sum_{k=1}^{M} \omega_{k} \operatorname{Re}\left[\frac{e^{K^{\prime}\left(\lambda_{r}+i \lambda_{k}\right)}}{\left(\lambda_{r}+i \lambda_{k}\right)^{2}} g_{0}\left(\lambda_{r}+i \lambda_{k}, v_{0}\right)\right],
\end{aligned}
$$

where $K^{\prime}=K N / F_{A},\left\{\omega_{k}\right\}_{k=1}^{M}$ is the weight vector of the numerical quadrature rule, $\left\{\lambda_{k}\right\}_{k=1}^{M}$ are the grid points, and $\lambda_{r}$ is a fixed parameter in the convergence region of $\Psi_{I_{N}}$.

By using the following integral representation of the expectation of square root of a random variable (Gatheral, 2006), where

$$
\mathbb{E}[\sqrt{X}]=\frac{1}{2 \sqrt{\pi}} \int_{0}^{\infty} \frac{1-\mathbb{E}\left[e^{-\lambda X}\right]}{\lambda^{3 / 2}} \mathrm{~d} \lambda,
$$

we derive the fair strike of the discrete volatility swap as follows

$$
\begin{aligned}
\mathbb{E}\left[\sqrt{V_{d}}\right] & =\sqrt{\frac{F_{A}}{N}} \mathbb{E}\left[\sqrt{I_{N}}\right] \\
& =\frac{1}{2} \sqrt{\frac{F_{A}}{N \pi}} \int_{0}^{\infty} \frac{1-\Psi_{I_{N}}(\lambda)}{\lambda^{3 / 2}} \mathrm{~d} \lambda \\
& \approx \frac{1}{2} \sqrt{\frac{F_{A}}{N \pi}} \sum_{k=1}^{M^{\prime}} \omega_{k}^{\prime}\left[\frac{1-g_{0}\left(\lambda_{k}, v_{0}\right)}{\lambda_{k}^{3 / 2}}\right],
\end{aligned}
$$

where $\left\{\omega_{k}^{\prime}\right\}_{k=1}^{M^{\prime}}$ is the weight vector of the numerical quadrature rule.

\section{Numerical tests}

In this section, we present the numerical tests for our recursive algorithms for pricing the discrete variance options and volatility swaps. The recursive algorithms are implemented under a variety of time-changed Lévy models, including the NIG and VG processes subordinated by a CIR process (NIG-CIR, VG-CIR models). The Gaussian quadrature is used for the evaluation of the "transition matrix". We demonstrate the performance of our numerical algorithm by comparing with the Monte Carlo simulation results as well as two other approximation methods. We also examine the sensitivity of variance put option prices and volatility swap fair strikes with respect to different model parameter values of the asset price models, including the correlation coefficient $\rho$ and volatility of the activity rate process $\epsilon$ under the Heston model (viewed as the geometric Brownian motion subordinated by a CIR process). 
In our numerical calculations, we adopted the set of parameter values of the NIG-CIR and VG-CIR models from Schoutens and Symens (2003), and the Heston model from Zhu and Lian (2011). The values of the model parameters are listed in Table 1. In addition, we assume that $d=0, S_{0}=1$ and $r=0.04$ and take $\Delta t=1 / 252$ for daily monitoring and $\Delta t=1 / 52$ for weekly monitoring. Our numerical experiments suggest that a Gaussian quadrature rule with 36 grid points with a finite range of $[-7,7]$ for $x$ is sufficient to approximate eq. (3.14) accurately. For the NIG-CIR and VG-CIR models, we further use a Gaussian quadrature rule with 100 points within the truncated range of $[0,5000]$ for $\lambda$ and approximate the infinite integrals with respect to $v$ by a Gaussian quadrature with 60 points within $[0,5]$. Finally, we find that $\lambda_{r}=4$ is an appropriate damping factor for evaluating the inverse Laplace transform in eq. (3.16).

\begin{tabular}{c|ccccccc}
\hline NIG-CIR & $\alpha$ & $\beta$ & $\delta$ & $k$ & $\theta$ & $\epsilon$ & $v_{0}$ \\
\hline parameter value & 18.48 & -4.84 & 0.469 & 0.539 & 1.575 & 1.877 & 1 \\
\hline \multicolumn{1}{l}{ VG-CIR } & $C$ & $G$ & $M$ & $k$ & $\theta$ & $\epsilon$ & $v_{0}$ \\
\hline parameter value & 11.99 & 25.85 & 35.53 & 0.602 & 1.556 & 1.999 & 1 \\
\hline \multicolumn{1}{c}{ Heston model } & $k$ & $\theta$ & $\epsilon$ & $\rho$ & $v_{0}$ & \\
\hline parameter value & 11.35 & 0.022 & 0.618 & -0.64 & 0.04 & & \\
\hline
\end{tabular}

Table 1: Parameter values of the time-changed Lévy models calibrated from market data.

Unlike the Heston model, we cannot adopt the Euler scheme directly in the Monte Carlo simulation of the sample paths under the general time-changed Lévy processes. For general pure jump processes, the jumps in the sample paths can be approximated by a number of Poisson processes, while for some special ones, we are able to simulate them by applying a time change to a Brownian motion with drift (Schoutens, 2003). For the NIG process, the large jumps are approximated by a number of independent Poisson processes representing different mean sizes of jumps and the small jumps by a Brownian motion. Asmussen and Rosinski (2001) establish a necessary condition for validity of the approximation of small jumps by a continuous Brownian motion. However, such a condition is not satisfied by the VG process, so a larger number of intervals are required for accurate approximation since the realized variance is sensitive to the percentage changes of the stock price. Therefore, the simulation time taken for the VG process is significantly longer than that of the NIG process. We followed the approach by Schoutens and Symens (2003) in the Monte Carlo simulation, where 100,000 sample paths for the NIG-CIR model and 10,000 paths for the VG-CIR model were simulated in our calculations. The recursive algorithms were coded in Mathematica to take advantage of its built-in Bessel functions. The computer programs were executed using a parallel algorithm in a multi-core Intel i7 PC.

\section{Assessment of numerical accuracy}

Performance under pure jump models with a CIR time change

In Table 2, we show the comparison of numerical accuracy for pricing at-the-money (ATM) undiscounted put options on daily monitored realized variance using four different methods: (i) recursive algorithm [eq. (3.16)], (ii) quadratic variation (QV) approximation where the discrete realized variance is approximated by quadratic variation, (iii) convexity correction 
method proposed by Keller-Ressel and Muhle-Karbe (KR-MK), (iv) Monte Carlo simulation method. Using the Monte Carlo simulation method as benchmark, we observe that the recursive algorithm provides highly accurate results compared to the other two approximation methods. In terms of computational efficiency, our recursive algorithm is quite effective with an average computational time of 120 seconds. The computational times for performing Monte Carlo simulation vary with the number of monitoring dates and can go up to hours for variance put options with longer maturity dates. Note that the computational (CPU) times for different numbers of monitoring dates $N$ in our algorithms are of the same order since the time required for the computation of the $N$-power of a $36 \times 36$ matrix $\left(h_{i j}\right)$ is very small compared to that of calculating the entries in the matrix [see eq. (3.14)]. With $N$ less than 40, the numerical errors are less than $1 \%$ across different strikes and monitoring dates under both the NIG-CIR and VG-CIR models. Similar comparison of numerical accuracy for pricing out-of-the-money (OTM) and in-the-money (ITM) put options on daily monitored realized variance using the same four methods is shown in Table 3. The CPU time for computing each price is more or less the same as that of the ATM case in Table 2 . Again, better numerical accuracy is achieved by the recursive algorithm compared to the two approximation methods.

For pricing put options on weekly sampled realized variance, a wider range of numerical integration in $v$ is required. From Table 4, we observe that the recursive algorithm produces pricing errors mostly less than $1 \%$ for small $N$. It again dominates the other methods in numerical accuracy. However, its performance for a relatively large $N$ appears to be not as good as the KR-MK correction method. While it is possible to further reduce the errors in our recursive algorithm results by choosing a finer grid of $v$, the CPU time would also be increased quite substantially.

Table 5 presents the numerical values of the fair strikes of the discrete volatility swaps priced under the NIG-CIR model and VG-CIR model that were computed by the recursive algorithm, QV approximation method and Monte Carlo simulation. Again, the recursive algorithm is seen to produce more accurate results, especially when $N$ is relatively small. We would like to remark that the KR-MK correction method cannot be applied to pricing volatility swaps since boundedness condition of the terminal payoff is not satisfied.

Though our recursive algorithms work well with high level of numerical accuracy and computational efficiency for short-maturity derivatives, one should be cautious in applying the recursive algorithms to pricing long-maturity (large $N$ ) options on discrete realized variance since the small numerical errors resulted from truncation and discretization may be accumulated quite significantly after multiplying the transition matrix recursively to a high order of $N$. Under such scenario, one has to take a denser set of grid points for the discretization and to enlarge the computational domain as well in order to achieve comparable level of accuracy. It may be advisable to use the quadratic variation approximation method for pricing long-maturity variance derivatives.

\section{Pricing behavior and sensitivity analysis}

We examine the pricing behavior of the discrete variance put option and volatility swap under various time-changed Lévy models by plotting the prices of the variance put options and fair strike prices of the volatility swaps against some chosen model parameters in these asset price models.

In Figures 1 and 2, we plot the undiscounted variance put option price and volatility swap fair strike against the model parameter $\alpha$ in the NIG-CIR model, respectively, where the 10-day daily monitored realized variance is used as the underlying. The 10-day at-the-money 
NIG-CIR model

\begin{tabular}{c|cccccc}
\hline$N$ (days) & 5 & 10 & 15 & 20 & 30 & 40 \\
\hline Strike (ATM) & 0.027807 & 0.028018 & 0.027927 & 0.027713 & 0.02836 & 0.028070 \\
\hline Recursive algorithm & 0.018819 & 0.016634 & 0.015212 & 0.01411 & 0.013307 & 0.012167 \\
CPU time (s) & 117.34 & 118.13 & 117.85 & 117.96 & 118.45 & 117.68 \\
\hline QV approximation & 0.018491 & 0.016418 & 0.014980 & 0.013857 & 0.013040 & 0.011980 \\
CPU time (s) & 1.15 & 1.07 & 1.06 & 1.01 & 0.94 & 0.89 \\
\hline KR-MK correction & 0.018922 & 0.016730 & 0.015420 & 0.014514 & 0.013322 & 0.012579 \\
CPU time (s) & 1.15 & 1.07 & 1.06 & 0.94 & 0.92 & 0.89 \\
\hline Monte Carlo & 0.018711 & 0.016627 & 0.015192 & 0.014072 & 0.013194 & 0.012087 \\
CPU time (s) & 1048.11 & 1850.68 & 2778.77 & 3714.03 & 6506.79 & 7401.58 \\
SE & 0.000067 & 0.000062 & 0.000057 & 0.000054 & 0.000052 & 0.000049 \\
\hline
\end{tabular}

VG-CIR model

\begin{tabular}{c|cccccc}
\hline Strike (ATM) & 0.027429 & 0.027502 & 0.027618 & 0.027752 & 0.028109 & 0.028239 \\
\hline Recursive algorithm & 0.019830 & 0.01708 & 0.015507 & 0.014452 & 0.013045 & 0.012056 \\
CPU time (s) & 113.64 & 113.75 & 114.51 & 115.35 & 115.74 & 113.97 \\
\hline QV approximation & 0.019588 & 0.016912 & 0.015334 & 0.014272 & 0.012986 & 0.012099 \\
CPU time (s) & 0.64 & 0.54 & 0.49 & 0.41 & 0.45 & 0.43 \\
\hline KR-MK correction & 0.019673 & 0.017005 & 0.015404 & 0.014307 & 0.012893 & 0.012037 \\
CPU time (s) & 0.62 & 0.55 & 0.47 & 0.40 & 0.45 & 0.41 \\
\hline Monte Carlo & 0.019835 & 0.017000 & 0.015580 & 0.014497 & 0.013002 & 0.012160 \\
CPU time (s) & 897.19 & 1802.00 & 2685.31 & 3567.90 & 5365.62 & 7148.78 \\
SE & 0.000224 & 0.000202 & 0.000190 & 0.000182 & 0.000169 & 0.000161 \\
\hline
\end{tabular}

Table 2: Comparison of numerical accuracy for pricing at-the-money (ATM) put options on daily monitored variance under pure jump models computed by the recursive algorithm, quadratic variation (QV) approximation, convexity correction method of Keller-Ressle and Muhle-Karbe, and Monte Carlo simulation. The computational (CPU) times are measured in units of second. In the Monte Carlo simulation, we used 100,000 paths for the NIG-CIR model, and 10,000 paths for the VG-CIR model. Here, SE stands for the standard error in the simulation.

strike for the variance put option is found to be 0.028018. It is known that the parameter $\alpha$ controls the tail behavior of the NIG distribution, so smaller $\alpha$ gives heavier tails. The respective plots show that the variance put option price is increasing in $\alpha$ while the fair strike of the volatility swap decreases in $\alpha$. This is expected since the tails of the NIG distribution become thinner as $\alpha$ increases. As a result, high asset returns or large realized variance would occur less likely.

We perform a similar sensitivity analysis of the discrete variance put option price and volatility swap strike with respect to the model parameter $C$ in the VG-CIR model. The 10-day ATM strike is found to be 0.027502. Madan et al. (1998) argue that $C$ provides the control over the kurtosis of the VG distribution. More precisely, the tail becomes thicker when $C$ increases. This conforms with the pricing behavior of the discrete variance put option and volatility swap shown in Figures 3 and 4 .

Lastly, we investigate the pricing behavior of the discrete variance put option and volatility swap under the Heston model, where the base Brownian motion is correlated with the CIR clock through the diffusion. In particular, we are interested in the sensitivity of the variance put price and volatility swap fair strike with respect to the correlation coefficient 
NIG-CIR model

\begin{tabular}{c|cccccc}
\hline$N$ (days) & 5 & 10 & 15 & 20 & 30 & 40 \\
\hline Strike (ITM) & 0.033369 & 0.033621 & 0.033351 & 0.033256 & 0.034035 & 0.033684 \\
\hline Recursive algorithm & 0.023507 & 0.021255 & 0.019701 & 0.018468 & 0.017662 & 0.016345 \\
QV approximation & 0.023230 & 0.021007 & 0.019430 & 0.018173 & 0.017346 & 0.016120 \\
KR-MK correction & 0.023760 & 0.021394 & 0.019981 & 0.019001 & 0.017706 & 0.016890 \\
Monte Carlo & 0.023453 & 0.021218 & 0.019650 & 0.018414 & 0.017489 & 0.016244 \\
SE & 0.000083 & 0.000077 & 0.000073 & 0.000069 & 0.000067 & 0.000063 \\
\hline Strike (OTM) & 0.022246 & 0.022414 & 0.022341 & 0.022171 & 0.022689 & 0.022456 \\
\hline Recursive algorithm & 0.014078 & 0.012180 & 0.010963 & 0.010006 & 0.009251 & 0.008322 \\
QV approximation & 0.013886 & 0.012014 & 0.010753 & 0.009794 & 0.009036 & 0.008176 \\
KR-MK correction & 0.014220 & 0.012252 & 0.011084 & 0.010283 & 0.009243 & 0.008609 \\
Monte Carlo & 0.014100 & 0.012214 & 0.010948 & 0.009986 & 0.009167 & 0.008274 \\
SE & 0.000052 & 0.000047 & 0.000043 & 0.000040 & 0.000038 & 0.000036 \\
\hline
\end{tabular}

VG-CIR model

\begin{tabular}{c|llllll}
\hline Strike (ITM) & 0.032915 & 0.033003 & 0.033141 & 0.033302 & 0.033727 & 0.033887 \\
\hline Recursive algorithm & 0.024321 & 0.021432 & 0.019749 & 0.018619 & 0.017112 & 0.016023 \\
QV approximation & 0.024158 & 0.021248 & 0.019548 & 0.018409 & 0.017051 & 0.016088 \\
KR-MK correction & 0.024261 & 0.021363 & 0.019635 & 0.018453 & 0.016931 & 0.016007 \\
Monte Carlo & 0.024443 & 0.021337 & 0.019808 & 0.018671 & 0.017050 & 0.016161 \\
SE & 0.000273 & 0.000249 & 0.000236 & 0.000227 & 0.000213 & 0.000205 \\
\hline Strike (OTM) & 0.021943 & 0.022002 & 0.022094 & 0.022202 & 0.022485 & 0.022591 \\
\hline Numerical Scheme & 0.015267 & 0.012836 & 0.011484 & 0.010537 & 0.009282 & 0.008422 \\
QV approximation & 0.015136 & 0.012751 & 0.011338 & 0.010387 & 0.009227 & 0.008453 \\
KR-MK adjustment & 0.015202 & 0.012822 & 0.011391 & 0.010412 & 0.009159 & 0.008409 \\
Monte Carlo & 0.015335 & 0.012824 & 0.011538 & 0.010600 & 0.009289 & 0.008504 \\
SE & 0.000176 & 0.000157 & 0.000146 & 0.000138 & 0.000127 & 0.000120 \\
\hline
\end{tabular}

Table 3: Comparison of numerical accuracy for pricing in-the-money (ITM) and out-of-themoney (OTM) put options on daily monitored variance under pure jump models computed by the recursive algorithm, quadratic variation (QV) approximation, convexity correction method of Keller-Ressel and Muhle-Karbe, and Monte Carlo simulation under daily monitoring. In the Monte Carlo simulation, we used 100,000 paths for the NIG-CIR model, and 10,000 paths for the VG-CIR model. Here, SE stands for the standard error in the simulation. The OTM strikes are taken to be $80 \%$ of the ATM strikes and $120 \%$ for the ITM strikes.

$\rho$ and volatility of variance $\epsilon$. Figures 5 and 6 show that both the discrete variance put option price and fair strike of volatility swap are insensitive to the correlation coefficient $\rho$. This is not surprising since the quadratic variation (continuous sampling limit of the discrete realized variance) of the time-changed Lévy process is seen to be independent of $\rho$. A similar phenomenon of insensitivity of the variance option price with respect to the correlation between the stock price process and variance process in the Heston model has also been reported in several earlier papers (Sepp, 2012; Drimus et al., 2014). It implies that when considering pricing derivative products on daily monitored realized variance one may just ignore the correlation between diffusion components. However, the impact of correlation does become significant when we consider derivative products on exotic realized variance, such as gamma swaps and corridor variance swaps (Zheng and Kwok, 2014a). On the other 
NIG-CIR model

\begin{tabular}{c|ccccc}
\hline$N$ (weeks) & 4 & 8 & 12 & 16 & 20 \\
\hline Strike (ATM) & 0.028267 & 0.028346 & 0.028832 & 0.029018 & 0.029200 \\
\hline Recursive algorithm & 0.015111 & 0.012905 & 0.012081 & 0.011319 & 0.010799 \\
QV approximation & 0.014382 & 0.012265 & 0.011545 & 0.011058 & 0.010793 \\
KR-MK correction & 0.014611 & 0.012654 & 0.011801 & 0.011386 & 0.011189 \\
Monte Carlo & 0.015068 & 0.012879 & 0.012123 & 0.011557 & 0.011259 \\
SE & 0.000058 & 0.000052 & 0.000050 & 0.000049 & 0.000048 \\
\hline Strike (ITM) & 0.033921 & 0.034015 & 0.034599 & 0.034822 & 0.035041 \\
\hline Recursive algorithm & 0.019583 & 0.017147 & 0.016255 & 0.015366 & 0.014758 \\
QV approximation & 0.018818 & 0.016473 & 0.015687 & 0.015113 & 0.014785 \\
KR-MK correction & 0.019106 & 0.016973 & 0.016020 & 0.015543 & 0.015307 \\
Monte Carlo & 0.019513 & 0.017128 & 0.016312 & 0.015688 & 0.015324 \\
SE & 0.000073 & 0.000066 & 0.000064 & 0.000069 & 0.000062 \\
\hline Strike (OTM) & 0.022614 & 0.022677 & 0.023066 & 0.023214 & 0.023361 \\
\hline Recursive algorithm & 0.010880 & 0.008976 & 0.008274 & 0.007664 & 0.007289 \\
QV approximation & 0.010198 & 0.008391 & 0.007786 & 0.007413 & 0.007224 \\
KR-MK correction & 0.010368 & 0.008673 & 0.007968 & 0.007644 & 0.007502 \\
Monte Carlo & 0.010856 & 0.008940 & 0.008298 & 0.007823 & 0.007594 \\
SE & 0.000043 & 0.000038 & 0.000037 & 0.000036 & 0.000034 \\
\hline
\end{tabular}

Table 4: Comparison of numerical accuracy for pricing put options on weekly monitored variance under pure jump models computed by the recursive algorithm, QV approximation, KR-MK correction, and Monte Carlo simulation. In the Monte Carlo simulation, we used 100,000 paths for the NIG-CIR model. Here, SE stands for the standard error in the simulation. The OTM (ITM) strikes are taken to be $80 \%$ (120\%) of the ATM strikes.

\begin{tabular}{l|ccccc}
\multicolumn{1}{l}{ NIG-CIR model } \\
\hline N (days) & 5 & 10 & 15 & 20 & 30 \\
\hline Recursive algorithm & 0.105724 & 0.120067 & 0.126671 & 0.132393 & 0.138035 \\
QV approximation & 0.107939 & 0.121797 & 0.129157 & 0.133923 & 0.139889 \\
Monte Carlo & 0.105879 & 0.120410 & 0.128103 & 0.133024 & 0.140125 \\
SE & 0.000524 & 0.000526 & 0.000527 & 0.000528 & 0.000537 \\
\hline
\end{tabular}

\begin{tabular}{c|ccccc}
\multicolumn{1}{l}{ VG-CIR model } & \multicolumn{1}{l}{} \\
\hline Recursive algorithm & 0.092962 & 0.111862 & 0.121129 & 0.128982 & 0.137155 \\
QV approximation & 0.091643 & 0.111988 & 0.122582 & 0.129188 & 0.137040 \\
Monte Carlo & 0.093534 & 0.112981 & 0.121593 & 0.128231 & 0.137675 \\
SE & 0.001696 & 0.001666 & 0.001648 & 0.001662 & 0.001677 \\
\hline
\end{tabular}

Table 5: Comparison of the numerical values of the fair strike prices of the daily monitored volatility swaps under pure jump models obtained from the recursive algorithm, QV approximation, and Monte Carlo simulation. In the Monte Carlo simulation, we used 100,000 paths for the NIG-CIR model, and 10,000 paths for the VG-CIR model. Here, SE stands for the standard error in the simulation.

hand, $\epsilon$ has some mild impact on the put option price and the fair strike of volatility swap. In fact, the variance put option price increases with an increase in $\epsilon$, whereas the fair strike of volatility swap is a decreasing function of $\epsilon$. 


\section{Conclusion}

We have proposed effective recursive algorithms for pricing discrete variance options and volatility swaps with short-maturity or low sampling frequency under a variety of timechanged Lévy processes. By employing the randomization formula, we obtain a recursive algorithm for computing the Laplace transform of the discrete realized variance. The resulting recursive algorithm admits a matrix representation which boils down to the calculation of a power of the transition matrix in which each entry in the matrix is the expectation of the transition probability with respect to different levels of instantaneous variance. By virtue of the efficient built-in algorithm in Mathematica for computing matrix power, we achieve a computational complexity that is almost constant across different maturities. The availability of the Laplace transform of the discrete realized variance enables us to calculate the prices of options on discrete realized variance and discrete volatility swaps using the standard inverse Laplace transform method. An intriguing feature of our recursive algorithm is that computational complexity of the algorithm is independent of the number of monitoring instants. However, in actual numerical implementation, denser grids are required to achieve the same level of numerical accuracy in numerical quadrature when the number of monitoring instants is higher, so the computational costs would increase.

We performed numerical calculations under the NIG-CIR, VG-CIR and Heston for pricing the discrete variance options and volatility swaps. We show that our recursive algorithms work particularly well in numerical accuracy for short-maturity variance derivatives compared to other approximation methods. The advantage of the recursive algorithms in numerical accuracy over other approximation methods is even more profound when we consider pricing under time-changed Lévy models with a nonzero diffusion component. In terms of computational efficiency, the recursive algorithms outperform the Monte Carlo simulation when we consider pricing discrete variance derivatives under time-changed Lévy models with jumps since the simulation of asset price paths with jumps is computationally demanding. In addition, we examine price sensitivity of the discrete variance option and volatility swap with respect to several model parameters in various time-changed Lévy models. 


\section{References}

[1] Aït-Sahalia, Y. (2004). Disentangling diffusion from jumps. Journal of Financial Economics, 74, 487-528.

[2] Asmussen, S. \& Rosinski, J. (2001). Approximations of small jumps of Lévy processes with a view towards simulation. Journal of Applied Probability, 38(2), 482-493.

[3] Baldeaux, J. (2012). Exact simulation of the 3/2 model. International Journal of Theoretical and Applied Finance, 15(5), 1-13.

[4] Barndorff-Nielsen, O.E. (1998). Processes of normal inverse Gaussian type. Finance and Stochastics, 2, 41-68.

[5] Barndorff-Nielsen, O. E., \& Shiriaev, A. N. (2010). Change of time and change of measure (Vol. 13). World Scientific.

[6] Bertoin, J. (1996). Lévy processes. Cambridge University Press, Cambridge.

[7] Broadie, M., \& Jain, A. (2008). The effect of jumps and discrete sampling on volatility and variance swaps. International Journal of Theoretical and Applied Finance, 11(8), 761-791.

[8] Brockhaus, O., \& Long, D. (2000). Volatility swaps made simple. Risk, 92-96, January.

[9] Bühler, H. (2006). Volatility markets: consistent modeling, hedging and implementation. PhD thesis, TU Berlin.

[10] Carr, P., Geman, H., Madan, D., \& Yor, M. (2002). The fine structure of asset returns: an empirical investigation. Journal of Business, 75, 305-332.

[11] Carr, P., Geman, H., Madan, D., \& Yor, M. (2003). Stochastic volatility for Lévy processes. Mathematical Finance, 13(3), 345-382.

[12] Carr, P., \& Wu, L. (2004). Time-changed Lévy processes and option pricing. Journal of Financial Economics, 71, 113-141.

[13] Carr, P., Geman, H., Madan, D., \& Yor, M. (2005). Pricing options on realized variance. Finance and Stochastics, 9, 453-475.

[14] Carr, P., \& Sun, J. (2007). A new approach for option pricing under stochastic volatility. Review of Derivatives Research, 10(2), 87-150.

[15] Carr, P., \& Lee, R. (2009). Volatility derivatives. Annual Review of Financial Economic$s, 1,319-339$.

[16] Carr, P., Lee, R., \& Wu, L. (2012). Variance swaps on time-changed Lévy processes. Finance and Stochastics, 16, 335-355.

[17] Cox, J., Ingersoll, J., \& Ross, S. (1985). Theory of the term structure of interest rates. Econometrica, 53, 385-408.

[18] Demeterfi, K., Derman, E., Kamal, M., \& Zhou, J. (1999). More than you ever wanted to know about volatility swaps. Goldman Sachs Quantitative Strategies Research Notes. 
[19] Drimus, G.G., Farkas, W., \& Gourier, E. (2014). Valuation of options on discretely sampled variance: a general analytic approximation. To appear in Journal of Computational Finance.

[20] Eberlein, E., Keller, U., \& Prause, K., (1998). New insights into smile, mispricing, and value at risk: the hyperbolic model. Journal of Business, 71, 371-406.

[21] Gatheral, J. (2006). The volatility surface. New York: Wiley finance.

[22] Fusai, G., \& Meucci, A. (2008). Pricing discretely Asian options under Lévy processes. Journal of Banking and Finance, 32, 2076-2088.

[23] Fusai, G., \& Recchioni, M.C. (2007). Analysis of quadrature methods for pricing discrete barrier options. Journal of Economic Dynamics and Control, 31(3), 826-860.

[24] Gatheral, J. (2006). The volatility surface. New York: Wiley finance.

[25] Geman, H., Madan, D., \& Yor, M. (2001). Time changes for Lévy processes. Mathematical Finance, 11, 79-96.

[26] Heston, S.L. (1993). A closed-form solution for options with stochastic volatility with applications to bond and currency options. Review of Financial Studies, 6, 327-343.

[27] Huang, J., \& Wu, L. (2004). Specification analysis of option pricing models based on time-changed Lévy processes. Journal of Finance, 59, pp. 1405-1440.

[28] Itkin, A., \& Carr, P. (2010). Pricing swaps and options on quadratic variation under stochastic time change models-discrete observations case. Review of Derivatives Research, $13(2), 141-176$.

[29] Jeanblanc, M., Yor, M., \& Chesney, M. (2009). Mathematical Methods for Financial Markets. Springer-Verlag, London.

[30] Kallsen, J., Muhle-Karbe, J., \& Voß, M. (2011). Pricing options on variance in affine stochastic volatility models. Mathematical Finance, 21(4), 627-641.

[31] Keller-Ressel, M., \& Muhle-Karbe, J. (2013). Asymptotic and exact pricing of options on variance. Finance and Stochastics, 17, 107-133.

[32] Kou, S.G. (2002). A jump-diffusion model for option pricing. Management Science, 48, 1086-1101.

[33] Lian, G.H., Chiarella, C., \& Kalev, P.S. (2014). Volatility swaps and variance options on discretely sampled realized variance. Journal of Economic Dynamics and Control, 47, 239-262.

[34] Little, T., \& Pant, V. (2001). A finite difference method for the valuation of variance swaps. Journal of Computational Finance, 5(1), 81-103.

[35] Lord, R., Fang, F., \& Oosterlee, C.W. (2008). A fast and accurate FFT-based method for pricing early-exercise options under Lévy processes. SIAM Journal on Scientific Computing, 30, 1678-1705.

[36] Madan, D.B., Carr, P.P., \& Chang, E.C. (1998). The variance gamma process and option pricing. European finance review, 2(1), 79-105. 
[37] Madan, D.B., \& Seneta, E. (1990). The Variance Gamma (VG) model for share market returns. Journal of Business, 63, 511-524.

[38] Merton, R.C. (1976). Option pricing when underlying stock returns are discontinuous. Journal of Financial Economics, 3, 125-144.

[39] Neuberger, A. (1994). The Log contract. Journal of Portfolio Management, 20(2), 74-80.

[40] Poteshman, A. (1998). Estimating a general variance model from option prices. Working paper, University of Chicago.

[41] Schoutens, W. (2003). Lévy Porcesses in Finance. John Wiley \& Sons Ltd, England.

[42] Schoutens, W., \& Symens, S. (2003). The pricing of exotic options by Monte-Carlo simulations in a Lévy market with stochastic volatility. International Journal of Theoreticl and Applied Finance, 6(8), 839-864.

[43] Sepp, A. (2008). Pricing options on realized variance in the Heston model with jumps in returns and volatility. Journal of Computational Finance, 11(4), 33-70.

[44] Sepp, A. (2012). Pricing options on realized variance in the Heston model with jumps in returns and volatility II: An approximate distribution of the discrete variance. Journal of Computational Finance, 16(2), 3-32.

[45] Sullivan, M.A. (2000). Pricing discretely monitored barrier options. Journal of Computational Finance, 3(4), 35-52.

[46] Swishchuk, A. (2004): Modeling of variance and volatility swaps for financial markets with stochastic volatilities, Wilmott Magazine, September Issue, Technical Article, 6472 .

[47] Windcliff, H., Forsyth, P.A., \& Vetzal, K.R. (2006). Pricing methods and hedging strategies for volatility derivatives. Journal of Banking and Finance, 30, 409-31.

[48] Umezawa, Y., \& Yamazaki, A. (2015). Pricing path-dependent options with discretely monitoring under time-changed Lévy processes. Applied Mathematical Finance, 22(2), 133-161.

[49] Yamazaki, A. (2014). Pricing average options under time-changed Lévy processes. Review of Derivatives Research, 17, 79-111.

[50] Zheng, W.D., \& Kwok, Y.K. (2014a). Closed form pricing formulas for discretely sampled generalized variance swaps. Mathematical Finance, 24(4), 855-881.

[51] Zheng, W.D., \& Kwok, Y.K. (2014b). Saddlepoint approximation methods for pricing derivatives on discretely sampled realized variance. Applied Mathematical Finance, $21(1), 1-31$.

[52] Zheng, W.D., \& Kwok, Y.K. (2014c). Fourier transform algorithms for pricing and hedging discretely sampled exotic variance products and volatility derivatives under additive processes. Journal of Computational Finance, 18(2), 1-28.

[53] Zheng, W.D., \& Kwok, Y.K. (2015). Pricing options on discrete realized variance with partially exact and bounded approximations. To appear in Quantitative Finance. 
[54] Zheng, W.D., \& Zeng, P.P. (2014). Transform methods for pricing exotic equity and volatility derivatives under the $3 / 2$ model. Working paper, Hong Kong University of Science and Technology.

[55] Zhu, S.P., \& Lian, G.H. (2011). A closed-form exact solution for pricing variance swaps with stochastic volatility. Mathematical Finance, 21(2), 233-256. 


\section{Appendix A: Proof of Lemma 1}

For the CIR process defined in eq. (2.2), one can derive the Laplace transform of the join$\mathrm{t}$ transition density of $v_{t}$ and $\int_{0}^{t} v_{s} \mathrm{~d} s$ by following the standard procedure of solving the characteristic function in the affine models. Specifically, we let

$$
\phi(\lambda, \eta)=\mathbb{E}\left[\exp \left(-\lambda v_{\tau}-\eta \int_{0}^{\tau} v_{s} \mathrm{~d} s\right) \mid v_{0}\right]=\exp \left(A(\tau)+v_{0} B(\tau)\right)
$$

one can obtain the Riccati equations for $A(\tau)$ and $B(\tau)$ (Jeanblanc et al., 2009). The functions $A(\tau)$ and $B(\tau)$ are given by

$$
\begin{aligned}
& A(\tau)=\frac{2 k \theta}{\epsilon^{2}} \ln \left(\frac{2 \delta e^{(\delta+k) \tau / 2}}{\epsilon^{2} \lambda\left(e^{\delta \tau}-1\right)+\delta\left(e^{\delta \tau}+1\right)+k\left(e^{\delta \tau}-1\right)}\right) \\
& B(\tau)=-\frac{\lambda\left(\delta+k+e^{\delta \tau}(\delta-k)\right)+2 \eta\left(e^{\delta \tau}-1\right)}{\epsilon^{2} \lambda\left(e^{\delta \tau}-1\right)+\delta\left(e^{\delta \tau}+1\right)+k\left(e^{\delta \tau}-1\right)}
\end{aligned}
$$

where $\delta=\sqrt{k^{2}+2 \epsilon^{2} \eta}$. To simplify the notations, we define

$$
\begin{gathered}
A_{1}=2 \delta e^{(\delta+k) \tau / 2}, B_{1}=\frac{1}{\epsilon^{2}\left(e^{\delta \tau}-1\right)}, C_{1}=\delta\left(e^{\delta \tau}+1\right)+k\left(e^{\delta \tau}-1\right), \\
D_{1}=-\left(\delta+k+e^{\delta \tau}(\delta-k)\right) v_{0}, E_{1}=-2 \eta\left(e^{\delta \tau}-1\right) v_{0} \text { and } \beta=\frac{2 k \theta}{\epsilon^{2}} .
\end{gathered}
$$

Suppressing the dependence on $\eta$, the joint characteristic function $\phi(\lambda)$ can be written as

$$
\phi(\lambda)=\exp \left(B_{1} D_{1}\right)\left(\frac{A_{1} B_{1}}{\lambda+C_{1} B_{1}}\right)^{\beta} \exp \left(\frac{B_{1}\left(E_{1}-B_{1} D_{1} C_{1}\right)}{\lambda+C_{1} B_{1}}\right) .
$$

On the other hand, let $f_{\mathrm{CIR}}\left(v, y, \tau ; v_{0}\right)$ be the joint transition density of $v_{\tau}$ and $\int_{0}^{\tau} v_{s} \mathrm{~d} s$ from $\left(v_{0}, 0\right)$ at time 0 to $(v, y)$ at time $\tau$, then the characteristic function can also be expressed as

$$
\begin{aligned}
\mathbb{E}\left[\exp \left(-\lambda v_{\tau}-\eta \int_{0}^{\tau} v_{s} \mathrm{~d} s\right) \mid v_{0}\right] & =\int_{0}^{\infty} \int_{0}^{\infty} e^{-\lambda v-\eta y} f_{\mathrm{CIR}}\left(v, y, \tau ; v_{0}\right) \mathrm{d} y \mathrm{~d} v \\
& =\int_{0}^{\infty} e^{-\lambda v} G_{\mathrm{CIR}}\left(v, \tau ; v_{0}, \eta\right) \mathrm{d} v .
\end{aligned}
$$

We may view $\exp \left(\left(A(\tau)+v_{0} B(\tau)\right)\right.$ as the Laplace transform of $G_{\mathrm{CIR}}\left(v, \tau ; v_{0}, \eta\right)$ on $v$. To recover the value of $G_{\mathrm{CIR}}$, we apply the inverse Laplace transform formula

$$
\begin{aligned}
\mathscr{L}^{-1}\left[p^{-\nu-1} e^{a / p}\right](x) & =\frac{1}{2 \pi i} \int_{\alpha-i \infty}^{\alpha+i \infty} e^{p x}\left(p^{-\nu-1} e^{a / p}\right) \mathrm{d} p \\
& =\left(\frac{x}{a}\right)^{\nu / 2} I_{\nu}(2 \sqrt{a x}),
\end{aligned}
$$

where $\nu>-1, a$ is a constant and $I_{\nu}$ is the modified Bessel function of the first kind of order $\nu$. Substituting $\phi(\lambda)$ into the above formula, we obtain 


$$
\begin{aligned}
& G_{\mathrm{CIR}}\left(v, \tau ; v_{0}, \eta\right) \\
= & \mathscr{L}^{-1}[\phi(\lambda)] \\
= & \mathscr{L}^{-1}\left[\exp \left(B_{1} D_{1}\right)\left(\frac{A_{1} B_{1}}{\lambda+C_{1} B_{1}}\right)^{\beta} \exp \left(\frac{B_{1}\left(E_{1}-B_{1} D_{1} C_{1}\right)}{\lambda+C_{1} B_{1}}\right)\right] \\
= & \left(A_{1} B_{1}\right)^{\beta} e^{\left(B_{1} D_{1}-C_{1} B_{1} v\right)} \mathscr{L}^{-1}\left[\left(\frac{1}{\lambda}\right)^{\beta} \exp \left(\frac{B_{1}\left(E_{1}-B_{1} D_{1} C_{1}\right)}{\lambda}\right)\right] \\
= & \left(A_{1} B_{1}\right)^{\beta} e^{\left(B_{1} D_{1}-C_{1} B_{1} v\right)}\left[\frac{v}{B_{1}\left(E_{1}-B_{1} D_{1} C_{1}\right)}\right]^{(\beta-1) / 2} I_{\beta-1}\left(2 \sqrt{B_{1}\left(E_{1}-B_{1} D_{1} C_{1}\right) v}\right) \\
= & 2 \exp \left(\epsilon^{2} k\left(v_{0}-v\right)-\frac{\left(1+e^{\delta} \tau\right)\left(v+v_{0}\right) \delta \epsilon^{2}}{e^{\delta \tau}-1}\right)\left[\frac{e^{-\delta \tau}\left(e^{\delta \tau}-1\right)^{2} v \epsilon^{4}}{v_{0} \delta^{2}}\right]^{\frac{\beta-1}{2}} \\
& {\left[\frac{e^{\frac{1}{2}(k+\delta) \tau} \delta}{\left(e^{\delta \tau}-1\right) \epsilon^{2}}\right]^{\beta} I_{\beta-1}\left(4 \sqrt{\frac{e^{\delta \tau} v_{0} v \delta^{2}}{\left(e^{\delta \tau}-1\right)^{2} \epsilon^{4}}}\right) . }
\end{aligned}
$$

\section{Appendix B: Parameter functions for the quadratic Gaus- sian OU process}

Let $\phi\left(t, s, z_{t} ; \lambda, \eta\right)=\mathbb{E}\left[\exp \left(\lambda v_{s}+\eta \int_{t}^{s} v_{u} \mathrm{~d} u\right) \mid \mathscr{G}_{t}\right]$, where $v_{t}=\left(z_{t}+p+q t\right)^{2}$ and $z_{t}$ is a Gaussian OU process: $\mathrm{d} z_{t}=\kappa\left(\theta-z_{t}\right) \mathrm{d} t+\sigma \mathrm{d} W_{t}$. Then, $\phi$ satisfies the following partial differential equation:

$$
\frac{\partial \phi}{\partial t}+\kappa(\theta-z) \frac{\partial \phi}{\partial z}+\frac{\sigma^{2}}{2} \frac{\partial^{2} \phi}{\partial z^{2}}+\eta(z+p+q t)^{2} \phi=0 .
$$

We look for a solution in the following exponential quadratic form:

$$
\phi\left(t, s, z_{t} ; \lambda, \eta\right)=\exp \left(H_{0}(t, s ; \lambda, \eta)+H_{1}(t, s ; \lambda, \eta) z_{t}+H_{2}(t, s ; \lambda, \eta) z_{t}^{2}\right) .
$$

Plugging the above exponential quadratic form into the governing equation yields the following Riccati system of ordinary differential equations for the parameter functions:

$$
\begin{aligned}
\frac{\partial H_{2}}{\partial t} & =2 \kappa H_{2}-2 \sigma^{2} H_{2}^{2}-\eta \\
\frac{\partial H_{1}}{\partial t} & =\kappa H_{1}-2 \sigma^{2} H_{1} H_{2}-2 \kappa \theta H_{2}-2(p+q t) \eta, \\
\frac{\partial H_{0}}{\partial t} & =-\kappa \theta H_{1}-\frac{\sigma^{2}}{2}\left(H_{1}^{2}+2 H_{2}\right)-(p+q t)^{2} \eta,
\end{aligned}
$$

with terminal conditions:

$$
H_{2}(s, s ; \lambda, \eta)=\lambda, H_{1}(s, s ; \lambda, \eta)=2(p+q s) \lambda, H_{0}(s, s ; \lambda, \eta)=(p+q s)^{2} \lambda .
$$


The solution to the above system is found to be

$$
\begin{aligned}
H_{2}(t, s ; \lambda, \eta)= & \frac{\lambda\left(\xi_{-} e^{-\zeta \tau}+\xi_{+}\right)+2 \eta\left(1-e^{-\zeta \tau}\right)}{\gamma_{+} e^{-\zeta \tau}+\gamma_{-}}, \\
H_{1}(t, s ; \lambda, \eta)= & \frac{4(p+q s) \lambda \zeta}{\gamma_{+} e^{-\zeta \tau / 2}+\gamma_{-} e^{\zeta \tau / 2}}+\frac{\kappa \theta}{\zeta} \frac{\left(\lambda \xi_{+}+2 \eta\right) e^{\zeta \tau / 2}-\left(\lambda \xi_{-}-2 \eta\right) e^{-\zeta \tau / 2}+4(\kappa \lambda-\eta)}{\gamma_{+} e^{-\zeta \tau / 2}+\gamma_{-} e^{\zeta \tau / 2}} \\
& +\frac{4 \eta p}{\zeta} \frac{\gamma_{+}\left(1-e^{-\zeta \tau / 2}\right)+\gamma_{-}\left(e^{\zeta \tau / 2}-1\right)}{\gamma_{+} e^{-\zeta \tau / 2}+\gamma_{-} e^{\zeta \tau / 2}}+\frac{4 \eta q}{\zeta} \frac{s\left(\gamma_{+}-\gamma_{-}\right)-t\left(\gamma_{+} e^{-\zeta \tau / 2}-\gamma_{-} e^{\zeta \tau / 2}\right)}{\gamma_{+} e^{-\zeta \tau / 2}+\gamma_{-} e^{\zeta \tau / 2}} \\
& -\frac{8 \eta q}{\zeta^{2}} \frac{\gamma_{+}\left(1-e^{-\zeta \tau / 2}\right)-\gamma_{-}\left(e^{\zeta \tau / 2}-1\right)}{\gamma_{+} e^{-\zeta \tau / 2}+\gamma_{-} e^{\zeta \tau / 2}}, \\
H_{0}(t, s ; \lambda, \eta)= & (p+q s)^{2} \lambda+\frac{\eta}{3}\left[(p+q s)^{3}-(p+q t)^{3}\right]-\frac{1}{4}\left[\xi_{+} \tau+2 \ln \frac{\gamma_{+} e^{-\zeta \tau}+\gamma_{-}}{2 \zeta}\right] \\
& +\int_{t}^{s}\left[\kappa \theta H_{1}(u, s ; \lambda, \eta)+\frac{\sigma^{2}}{2} H_{1}(u, s ; \lambda, \eta)^{2}\right] \mathrm{d} u,
\end{aligned}
$$

where $\tau=s-t$, and

$$
\begin{aligned}
\zeta & =2 \sqrt{\kappa^{2}-2 \sigma^{2} \eta} \\
\xi_{ \pm} & =\zeta \mp 2 \kappa, \\
\gamma_{ \pm} & =\xi_{ \pm} \pm 4 \sigma^{2} \lambda .
\end{aligned}
$$




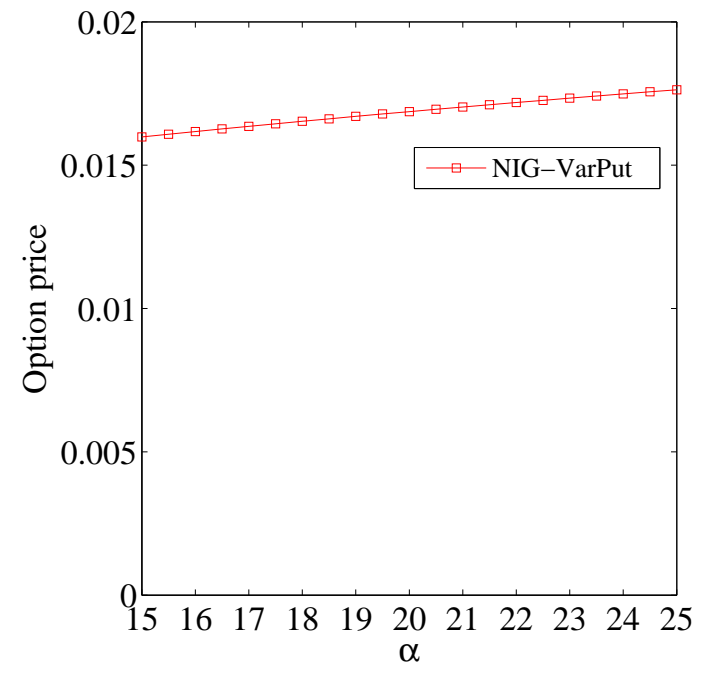

Figure 1: Plot of the price of the 10-day put option on daily monitored realized variance against the model parameter $\alpha$ in the NIG-CIR model. The at-the-money strike is taken to be 0.028018 .

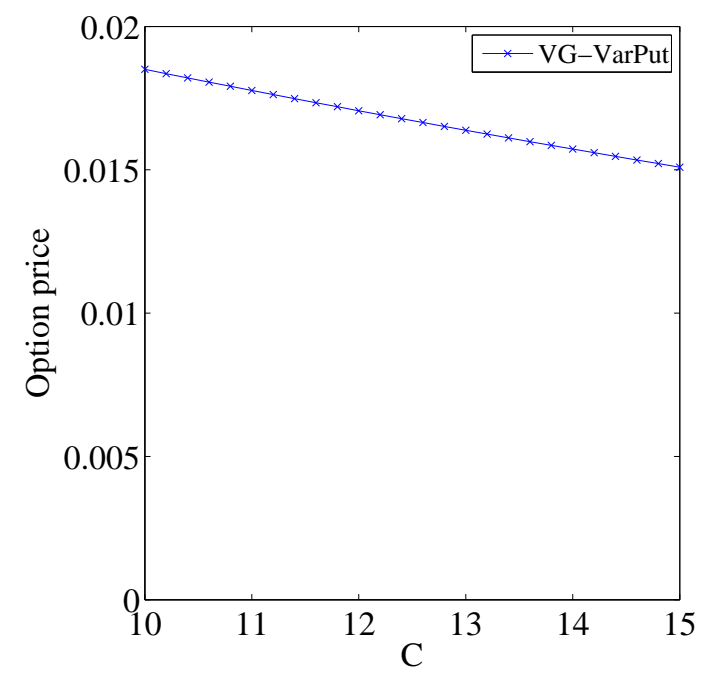

Figure 3: Plot of the price of the 10-day put option on daily monitored realized variance against the model parameter $C$ in the VG-CIR model. The at-the-money strike is taken to be 0.027502 .

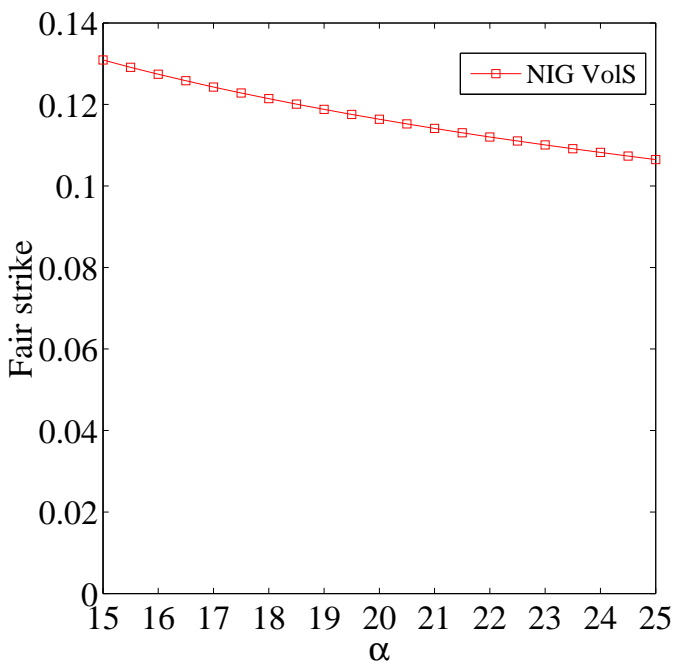

Figure 2: Plot of the fair strike of the 10-day daily monitored volatility swap against the model parameter $\alpha$ in the NIGCIR model.

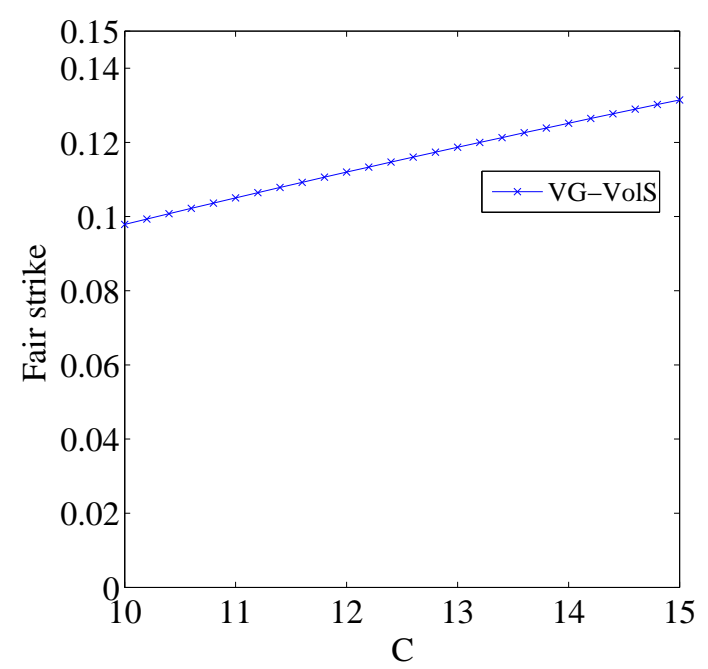

Figure 4: Plot of the fair strike of the 10-day daily monitored volatility swap against the model parameter $C$ in the VGCIR model. 


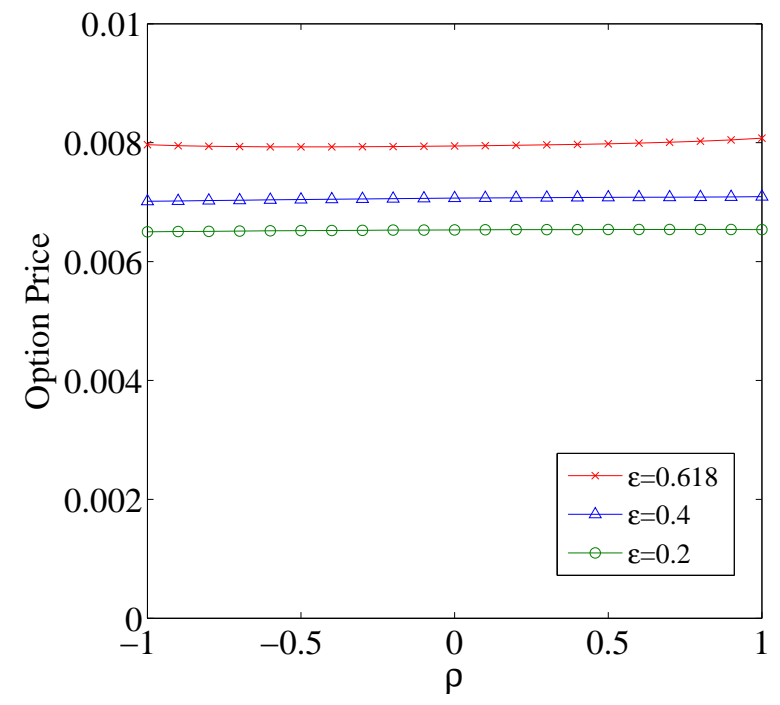

Figure 5: Plot of the price of the 10-day put option on daily monitored realized variance against the correlation coefficient $\rho$ given three different values of $\epsilon$ in the Heston model. The at-the-money strike is taken to be 0.03640 .

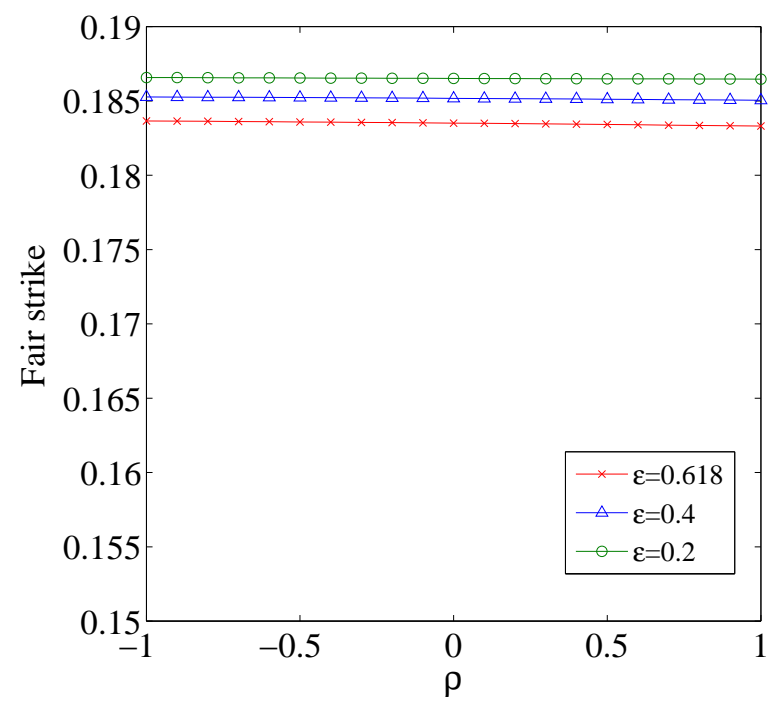

Figure 6: Plot of the fair strike of the 10-day daily monitored volatility swap against the correlation coefficient $\rho$ given three different values of $\epsilon$ in the Heston model. 